\title{
Fortsetzung des Wandels unter Präsident Mbeki (1999-2009)
}

\subsection{Südafrika zum Ende der 1990er Jahre - Stockender Wandlungsprozess}

Sowohl seitens des ANC als auch der SACP, und zwar vor wie nach den Wahlen 1999, wurde die fortdauernde Bedeutung des RDP hervorgehoben und es kam zu einer auch vom COSATU unwidersprochenen und geradezu zelebrierten ${ }^{461}$ Neubestätigung bzw. Wiederbestätigung des RDP („reaffirmation of the RDP ${ }^{{ }^{4} 62}$ ) durch die Tripartite Alliance.

In Veröffentlichungen und Verlautbarungen des Department of Trade and Industry (DTI) und auch in denen der Botschaften im Ausland, z. B. in Bonn, wurde der Fortbestand und die wichtige Bedeutung des RDP für die Umwandlung Südafrikas stets unterstrichen. In einer Publikation wurde sogar betont, dass das RDP in das Programm für „Wachstum, Beschäftigung und Umverteilung" (GEAR) „eingebettet“ sei, um damit „die sozialen Ungleichheiten im Land möglichst rasch abzubauen und den Lebensstandard aller Südafrikaner zu verbessern ${ }^{4463}$, womit ja wohl - indirekt - zugegeben wird, dass die sozialen Ungleichheiten immer noch bestehen und es um den Lebensstandard der südafrikanischen Unterschichten ${ }^{464}$, jedenfalls Ende der 1990er, bzw. zu Beginn der zweiten Legislaturperiode der ANC-Regierung, immer noch nicht so gut bestellt war, wie erhofft.

461 Nach den vielen Differenzen über das „ergänzende“ und wirklich teilweise das RDP ablösende Programm GEAR

462 McKinley (2001), S. 74.

463 DTI \& Südafrikanische Botschaft Bonn/Berlin (1997), S. 5.

464 Vor allem der größte Teil der völlig verarmten Unterschicht, deren Angehörigen schon deshalb oft „irrelevant“ wurden und noch werden („considered irrelevant“ (Human (1998), S.206)), zumal sie kaum oder gar nicht als Konsumenten auf einem Markt auftreten.

(C) Springer Fachmedien Wiesbaden GmbH, ein Teil von Springer Nature 2019

M. T. P. Sprenger-Menzel, Von der Apartheidsgesellschaft zur Rainbow

Nation, Globale Gesellschaft und internationale Beziehungen,

https://doi.org/10.1007/978-3-658-27507-5_5 
Während die südafrikanische Regierung schneller Ergebnisse bedurfte, um sich die Unterstützung der Massen bzw. ihre zukünftigen Wähler zu erhalten und die Demokratie zu sichern, war und ist zur Finanzierung und Durchführung der wirtschafts- und sozialpolitischen Reformprogramme ein hohes, das Bevölkerungswachstum (Geburten plus Wachstum durch Zuwanderung aus den Nachbarländern) ü b e rste ige nde s Wirtschaftswachstum ${ }^{465}$ nötig, um Arbeitsplätze zu schaffen, die mangelnde Infrastruktur in den vornehmlich von Schwarzen bewohnten Teilen des Landes auszubauen, Wohnungen zu bauen und das Bildungswesen ausweiten und verbessern zu können.

Die Regierung Südafrikas muss hierzu gerade jene Leistungsträger mit immer mehr öffentlichen Abgaben belasten, die sie für den wirtschaftlichen Auf- und Ausbau des Landes am dringendsten benötigt, nämlich die (zur Zeit noch meist weißen) Angehörigen des primary market, der ohnehin seit Jahren von einem außergewöhnlich starken brain drain ausgeblutet wird (allein 1996 haben 13 Prozent der 1.200 Spitzenmanager mit ihren Familien das Land verlassen). Diese wachsende Belastung durch Steuern und Sozialabgaben - bei gleichzeitig sinkender Lebensqualität für diese Bevölkerungsgruppe - ist nötig, um damit die Masse der Bevölkerung, die wegen Arbeitslosigkeit oder wegen geringen Einkommens und Vermögensbesitzes wenig oder überhaupt keine Steuern zahlt (hauptsächlich schwarze Südafrikaner), motivieren, unterstützen und fördern zu können.

Nach Angaben des Handels- und Industrieministeriums (DTI) mussten sich die (immer noch hauptsächlich weißen) Steuerzahler des Landes auf 16 neue Steuern ${ }^{466}$ einstellen, welche die Regierung in Kürze einzuführen plante. Weiter erschwert wurde der Transformationsprozess zusätzlich dadurch, dass in Südafrika inzwischen de facto eine hohe Kriminalität, ja sogar ein krimineller Ausnahmezustand herrschte, wie der südafrikanische Minister für Sicherheitsangelegenheiten, Sydney Mufamadi, in einem Vortrag über Investitionen und Sicherheit vor potentiellen deutschen und ausländischen Investoren auf einer von der südafrikanischen Botschaft in Bonn organisierten „investment conference" am 5. Juni 1997 in Bad Honnef einräumen musste. So hatten allein von April 1994 bis April 1997 über 30.000 Polizisten den Dienst quittiert. Von den neu eingestellten Polizeibeamten besaß fast ein Drittel keinen Führerschein, und allein in einem Jahr (1995) musste "gegen 2.848 Polizeibeamte wegen krimineller Delikte ermittelt werden, darunter Korruption, Betrug

465 Ein Beispiel: Im Jahre 1990 lag das Bevölkerungswachstum bei nur 2,3 Prozent, doch schon dieses geringe Bevölkerungswachstum (in den letzten Jahren lag es etwas höher) bewirkte eine Senkung des Bruttoinlandsproduktes pro Kopf 1990 um 11,3 Prozent (vgl. auch Eggers (1997), S. 249).

466 Hervorhebung im Original. 
und vor allem Mord. ${ }^{4467}$ Bei gleichzeitig fehlenden 6.228 Justizvollzugsbeamten (Gefängnisaufsehern) waren die Gefängnisse zu 150 Prozent überbelegt und viele Häftlinge wurden wegen Überfüllung vorzeitig entlassen. Die südafrikanische Ausbruchsrate ist besonders hoch ${ }^{468}$. Von April 1994 bis Dezember 1996 hat ein Drittel aller Staatsanwälte den Dienst quittiert, und die jungen Staatsanwälte haben im Durchschnitt weniger als zwei Jahre Berufserfahrung. ${ }^{469}$

In den Jahren seit 1994 hat man zwar viele Verwaltungsbeamte in den (oft) vorzeitigen Ruhestand geschickt, doch die Effizienz und Effektivität der öffentlichen Verwaltung in Südafrika liegt zunehmend im Argen. Eine Umsetzung der Ziele des RDP ist aber ohne eine funktionierende öffentliche Verwaltung ebenso wenig erreichbar wie die Erhöhung des Wirtschaftswachstums auf - mindestens - 6 und mehr Prozent. Hier schlummert ein für die sozioökonomische Transformation gefährliches Konfliktpotential, das - trotz aller bisher erbrachten Leistungen - die neue Demokratie in Südafrika bis heute gefährden kann, zumal wegen der immer offener zutage tretenden negativen Auswüchse der öffentlichen Verwaltung.

Die Ungeduld des Volkes über die fehlende Erbringung von üblichen Dienstleistungen des öffentlichen Dienstes im öffentlichen Personennahverkehr, bei der Feuerwehr und Polizei, in den Schulen und offenbar überall in den Kommunalverwaltungen (,missing service deliver ${ }^{\mathrm{C}_{470}}$ ) wächst, und, nach ganz aktuellen Umfragen, ist das Vertrauen in die Staatsorgane, in die öffentliche Verwaltung und auch in die Polizei sehr stark gesunken ${ }^{471}$.

467 Vgl. z. B. Der Spiegel, 01.09. 1997, S. 158 und Scarborough (1997), S. 12.

468 Vgl. z. B. die kleine stichprobenartige Übersicht zur Kriminalität in Südafrika, in deren letzter Sparte auf der dritten Seite (Jahr 1997) wo nach 937 Ausbrüchen bis Anfang Dezember, die Business Day vom 12.12.1997 von der Gefängnisaufsicht vermutet, dass man die Situation (jetzt wieder) ,unter Kontrolle“ habe („Situation in SA Prisons 'under control' despite 937 Escapes“).

469 Vgl. SAIRR (1997).

470 Vgl. Mc Lennan (2009) und auch Hemson, Carter \& Karuri-Sebina (2009).

471 Diese Einschätzungen beruhen offenkundig auf langjährigen Erfahrungen bzw. Enttäuschungen der Steuer- und Gebührenzahler Südafrikas, welche die schleppend, fehlerhaft oder gar nicht erbrachten Dienstleistungen der öffentlichen Verwaltung und auch die fehlende Arbeitsmotivation eines Großteils der Mitarbeiter seit vielen Jahren erleben und dies leid sind, wie man an den Einschätzungen aus der Tabelle 9 erkennen kann. Wie es um viele Bereiche des öffentlichen Dienstes selbst in Großstädten Südafrikas bestellt ist, beschrieb in seiner Dissertation Dr. Dirk Asendorpf, der - sieben Jahre nach Beginn des RDP und in der zweiten Legislaturperiode des ANC unter Präsident Mbeki - u. a. die Arbeits- und Einsatzqualität der Feuerwehr, die Polizei und Dienststellen der allgemeinen Verwaltung am Beispiel der Universitätsstadt (Walter-Sisulu-University) und Distrikthauptstadt des Distriktes (des Bezirkes) OR Tambo in der Provinz Eastern 
Nach den Ergebnissen einer aktuellen Umfrage, die das renommierte Institute for Security Studies (ISS) durchführte, glaubt die Hälfte aller Südafrikaner, dass „alle“ (,all“) oder „,die meisten“ („most“) Polizeibeamten Südafrikas in Korruption verstrickt seien. ${ }^{472}$ Nach anderen, im März 2019 veröffentlichten Umfragen trauen zwei Drittel der Polizei „ganz und gar nicht“ oder „überhaupt nicht“ („not at all“) oder nur „ein wenig“ bzw. „etwas“(„a little“).

Tab. 9 Wem vertrauen Südafrikaner "ganz und gar nicht“ oder nur „etwas “473

\begin{tabular}{lc}
\hline Wie sehr trauen Sie; wem trauen Sie: & $\begin{array}{c}\text { „überhaupt nicht/gar nicht“ } \\
\text { „nur etwas/ein bisschen“ }\end{array}$ \\
\hline Traditional leaders (z.B. Häuptlingen) & 43 \\
\hline Religious leaders (Geistlichen im weitesten Sinne) & 43 \\
\hline Courts (Gerichten) & 46 \\
\hline Judges (Richtern) & 49 \\
\hline South African Revenue Service (SARS) (Steuerbehörden) & 50 \\
$\begin{array}{l}\text { The Public Protector (Volksanwalt mit richterlicher } \\
\text { Unabhängigkeit) }\end{array}$ & 50 \\
$\begin{array}{l}\text { The Premier of this province (dem Landesminister- } \\
\text { präsidenten) }\end{array}$ & 56 \\
\hline The President (dem Präsidenten Südafrikas) & 57
\end{tabular}

Cape, Mthata (früher als „Umtata“ Hauptstadt der Transkei), in Fallstudien untersucht hat. Totalausfälle der Feuerwehr, die wegen falscher und undichter Schläuche und fehlender Einsatzübungen gar nicht einsatzfähig ist, Polizei, die ihre Beamten zu deren Schutz nicht in "hostile areas" (Asendorpf (2001), S. 238) sendet, Kommunalbeamte, die morgens ihre Jacken sichtbar aufhängen und dann, nach langen Stunden in Bars oder Geschäften, nachmittags nur noch „zum Ausstempeln“kurz an ihren Arbeitsplatz zurückkehren, und andere unglaubliche Umstände und Nachlässigkeiten, die auch nicht geahndet wurden, werden von Asendorpf sehr detailliert beschrieben (vgl. Asendorpf, insbesondere Kap. 12 „In der Warteschlange - Dienstleistungen als Mangelware“), z. B. die Abschnitte „Es brennt, und die Feuerwehr guckt zu“ oder „Schlafen verboten - Ein Besuch im Verwaltungshochhaus" (in welchem die Mehrzahl der vom Steuerzahler besoldeten Beamten tatsächlich (fast) den ganzen Tag gar nicht anwesend ist, und Bürger teilweise jahrelang auf Bescheide oder Geldauszahlungen warten), S. 230-256. Es dürfte sofort einsichtig sein, dass eine nicht ordentlich funktionierende öffentliche Verwaltung, z. B. wegen zu langer Bearbeitungsdauern bei Bauanträgen, Investitionsvorhaben etc. negativ auf das in Südafrika so dringend benötigte Wirtschaftswachstum (auch zur Finanzierung des RDP) auswirken könnte (vgl. auch Creamer (2010), S. 219).

472 Vgl. Faull (2019).

473 Tabelle in enger Anlehnung an die Tabelle „How much do you trust ..." in ebd., alle Übersetzungen stammen vom Verfasser). 


\begin{tabular}{lc}
\hline Wie sehr trauen Sie; wem trauen Sie: & $\begin{array}{c}\text { „überhaupt nicht/gar nicht“ } \\
\text { „nur etwas/ein bisschen“ }\end{array}$ \\
\hline $\begin{array}{l}\text { The Independent Electoral Commission (IEC) } \\
\text { (Unabh. Wahlkommission) }\end{array}$ & 58 \\
\hline $\begin{array}{l}\text { The African National Congress (ANC) (die Regierungs- } \\
\text { partei ANC) }\end{array}$ & 60 \\
\hline $\begin{array}{l}\text { Parliament (Staatsparlament) } \\
\text { Your local government council (Ihrem örtlichen } \\
\text { Gemeinderat) }\end{array}$ & 65 \\
\hline $\begin{array}{l}\text { Police (Polizei) } \\
\text { Opposition political parties (politische Oppositions- } \\
\text { parteien) }\end{array}$ & 65 \\
\hline
\end{tabular}

Die in Tabelle 9 stehenden Zahlen, z. B. auch zum fehlenden Vertrauen in das Justizwesen, dem rund die Hälfte der Befragten nicht traut, hätte man vor dreißig oder vierzig Jahren, zur Zeit der Apartheid sofort verstanden, weil damals der schwarzen Bevölkerung der Zugang zum formalen Rechtssystem verweigert wurde, so dass es zu aktiven Ablehnungen und Misstrauen kommen musste, weil Richter und Gerichte als Teil des staatlichen Unterdrückungssystems während der Apartheid betrachtet wurden, was noch in den Tagen zu Beginn der Umsetzung des RDP in Umfragen bestätigt worden ist. ${ }^{474}$

Dass aber - beinahe volle 25 Jahre nach Beginn der Einführung und Umsetzung des RDP - enttäuschende Umfragewerte wie jene vorkommen, die in Tabelle $9 \mathrm{zu}$ finden sind, ist angesichts der Tatsache, dass der ANC, und dies ganz im Geiste und Sinne des RDP, den Menschen und dessen Bedürfnisse stets besonders in den Vordergrund rückte ${ }^{475} \mathrm{bzw}$. seit etwa 1990 ein anzustrebendes besseres Leben für alle (,, a better life for all“) ${ }^{476}$ versprach, tatsächlich als Alarmzeichen zu betrachten

474 Van der Merwe \& Mbebe (1994), S. 2.

475 Vgl. auch Dressel \& Neumann (2001), S. 152. Die Losung bzw. die Forderung „A better life for all“ stand auch fast in jedem ANC-Dokument der frühen 1990er Jahre (vgl. z. B. ANC (1994)) zu lesen.

476 Diese Losung bzw. Formel („A better life for all“) fehlte in den frühen 1990er Jahr in keiner Rede Präsident Mandelas oder anderer Parteiführer des ANC und fand sich auch in fast jeder Publikation des ANC, oft auch bzw. zusätzlich auf der Außen- oder Rückseite der Dokumente (vgl. ANC (1990), ANC (1991), ANC (1992), ANC (1994a), ANC (1994b), ANC (1994b) und auch ANC (2007)), schließlich auch in einer im Wahlkampf verteilten, vom ANC und Nelson R. Mandela gemeinsam herausgegebenen Broschüre an die „Fellow South Africans“ (ANC/Mandela (o. J.), S. 2). 
und aus Sicht der unzufriedenen Bevölkerung, wie es sich auch in Tabelle 9 manifestiert, nicht hinnehmbar.

Die mangelnde Qualität des öffentlichen Dienstes und bei der Aufrechterhaltung der inneren Sicherheit Südafrikas zog einige Zeit lang selbstverständlich auch negative Folgen für den außenpolitischen und - vor allem auch - den außenwirtschaftlichen Status Südafrikas als Investitionsland nach sich.

Dabei bedurfte Südafrika dringend ausländischer Investoren, und genau deshalb warb Südafrika im Juni und Juli 1997 in mehreren (oben schon angesprochenen) "Investitionskonferenzen“ (,Made in South Africa - Investment Conference“), für deren Ausrichtung man keine Ausgaben und Mühen scheute ${ }^{477}$, in mehreren Ländern der Europäischen Union, vor allem in der Bundesrepublik Deutschland und im Vereinigten Königreich, um Investoren bzw. Unternehmensgründer.

Nach dem Austritt Südafrikas aus dem Commonwealth hatte in der jungen „Republik Südafrika“ die Inflationsrate in den ganzen 1960er Jahren vergleichsweise niedrig gelegen (im Durchschnitt bei etwa 2,4 Prozent). Sie stieg in den 1970er Jahren mit den Ölkrisen (1973 und 1979), dem Zusammenbruch des Bretton Woods-Systems (1971-1973) und aus weiteren, gerade auch globalen ökonomischen und politischen Gründen, nicht nur international, sondern auch konkret in Südafrika an (im Durchschnitt rund 9,8 Prozent) und - im Durchschnitt der 1980er Jahre - sogar auf rund 14 Prozent an. ${ }^{478}$

Abgesehen von der hohen Arbeitslosenquote waren die aktuellen Wirtschaftskennzahlen Südafrikas zu Anfang der 1990er Jahre relativ positiv. Doch angesichts der immensen Aufgaben offenkundig nicht positiv genug (eine einstellige Inflationsrate eine sinkende Staatsverschuldung in Höhe von rund 5 Prozent des Bruttoinlandsproduktes) und in den späten 1990er Jahren gingen wegen zu geringen Wachstums, hoher Importe und leider eher kapital- sowie energieintensiver Investitionen ohne die erwartete gleichzeitige Schaffung neuer Arbeitsplätze, Millionen Arbeitsplätze verloren. ${ }^{479}$

Wegen des unverändert hohen Bevölkerungswachstums von fast 3 Prozent und der hohen Immigrationszahlen (in letzter Zeit häufen sich Klagen in südafrikanischen Zeitungen über die „illegal immigrations“, und in wissenschaftlichen Aufsätzen

477 In Deutschland fand die Konferenz im Gästehaus der Bundesregierung auf dem Petersberg statt, zu dem die Teilnehmer eigens mit Bussen transportiert worden waren. Es gab tatsächlich sehr gute Vorträge, Infomationsmaterial in Afrikaans, Deutsch und Englisch und Möglichkeit zum Austausch mit Unternehmern, Beamten des DTI und Vertretern der Wirtschaftsabteilung der Botschaft von Südafrika (vgl. auch DTI (1997)).

478 Vgl. Michl (1988), S. 26.

479 Vgl. Bond \& Erion (2009), S. 355. 
wurde schon vor über zwanzig Jahren über eine „Invasion der Fremden“ („,an alien invasion in South Africa") diskutiert ${ }^{480}$ ) ist eine spürbare Anhebung des allgemeinen Lebensstandards wohl nur bei Wachstum von mindestens 6 Prozent, besser mehr Prozent erreichbar, wenn weitere tiefgreifende Redistributionsmaßnahmen, vor allem weitere Steuererhöhungen, vermieden werden sollen.

480 Vgl. hierzu Maharaj/Rajkumar (1997). Auffällig sind auch die seit den Wahlen 1994 stetig zunehmenden Artikel mit den Stichworten „illegal aliens“ „illegal immigration“, "repatriation" (schon im ersten Regierungsjahr Präsident Mandelas 1994 allein nach Mosambik 60.000 Personen) in vielen südafrikanischen Zeitungen. In der "Citizen“ findet sich die Meldung, dass im Haushaltsjahr 1996/97 mehr als 7,57 Millionen Rand „on repatriating or deporting 180.713 illegal aliens" ausgegeben wurden (Citizen, 10.04.1997: „Illegal Aliens cost R7,57M“). Vgl. allgemein zum Thema auch Reitzes (1995a); Reitzes (1995b); Carim (1996). Xavier Carim ist Research Fellow an dem Centre for Southern African Studies an der UWC. Vgl. zur Thematik internationale Migration, Flucht und Asyl allgemein Nuscheler (2004), insbesondere den Teil I: „Migration und Flucht“, S. 21-60 und die Artikel im Sammelband Bekker/Carlton (1996). Zu dem in Südafrika schon wenige Jahre nach der Erlangung der Demokratie immer wichtiger werdenden und damit sogar schon einige der bis dato ohnehin bestehenden wirtschaftlichen, sozialen und demographischen Probleme weiter verstärkenden Thema „Immigration in Südafrika“ vgl. z. B. den Bericht von der südafrikanischen „Abwehrfront“ Grills (1998) und auch Goebel (1999), S.64-136 und S.307-332. Mindestens schon Anfang des III. Jahrtausends waren die Begriffe „Fremdenfeindlichkeit“, nicht nur im Sinne einer „Fremdenangst“ (Angst vor Fremden, insbesondere ausländischen Immigranten aller Art und Herkunft), meist „xenophobia“ genannt, bereits in aller Munde und tauchten fast täglich in Zeitungsmeldungen auf, anfänglich, oft noch als „Randthema“, auch in streng wissenschaftlicher Literatur (vgl. z. B. Sichone (2004), S. 315 und im Handbuch James Chidesters zur „sozialen Kohäsion“ („Whatholdsustogether (sic!). Social cohesion in South Africa“ (vgl. Chidester, Hadland \& Prosalendis (2004), passim). Wenige Jahre später. jedoch schon vor über einem Jahrzehnt, tauchte der Begriff „Xenophobia“ dann schon in Handbüchern zur Alltagssprache bzw. zu wichtigen neuen südafrikanischen „Schlüsselwörtern“, wie z. B. „New South African Keywords“ (vgl. Sichone (2009) auf (vgl. Shepherd \& Robins (2008)). Die starke Immigration nach Südafrika hielt zwischen 1994 und 2019 an und es kam auch immer wieder zu fremdenfeindlichen Ausschreitungen und Unruhen (vgl. z. B. FAZ vom 21.02.2001: „Soziale Unrast in Südafrika“). Selbst politisch Verfolgte wurden schnellstens wieder in ihre Heimatländer deportiert, z. B. 2007 allein 200.000 Mosambikaner, weil Südafrika bei der Anerkennung von Asylanträgen sehr restriktiv verfuhr und z. B. trotz hunderttausender Flüchtlinge zwischen 2004 und 2006 nur 246 Personen als „politische Flüchtlinge“ bzw. „politisch Verfolgte anerkannte (Vgl. Kohnert, der über „neuen Nationalismus und Fremdenfeindlichkeit“ im subsaharischen Afrika berichtet; Kohnert (2008), S. 2). 
Im November 1997 stellte der Gouverneur der Südafrikanischen Zentralbank (SARB), Dr. Chris Stals, für das Jahr 1998 einen weiteren Abbau von Devisenkontrollen in Aussicht und kündigte „other sweeping reforms ${ }^{{ }^{c 481}}$ an.

Im Dezember 1997 veröffentlichte Finanzminister Trevor Manuel in einem Dokument die mittelfristigen Haushaltsplanungen für die kommenden drei Jahre. Ab 1998 sollte mit einem Medium Term Expenditure Framework (MTEF) das bisherige Haushaltsverfahren geändert werden, um der südafrikanischen Regierung damit einen (festeren) „Zugriff auf die Ausgabenplanung im Einklang mit den mittelfristigen strategischen und politischen Zielen ${ }^{\text {“482 }}$ geben zu können.

Ein für die zweite Jahreshälfte 1998 - und schon spürbarer für 1999 - nicht nur von der Regierung stets prophezeite und längst erwartete Aufschwung trat wegen des wieder wachsenden Vertrauens der Wirtschaft, insbesondere auch bei den schon lange auf den Aufschwung oder wenigstens dessen Anzeichen wartenden börsennotierten Unternehmen in Südafrika ${ }^{483}$, dann auch tatsächlich ein, wie wichtige volkswirtschaftliche Indikatoren zeigten.

Mitglieder des ANC und des COSATU waren in früher den Weißen vorbehaltenen Spitzenpositionen in Wirtschaft, Politik und öffentlicher Verwaltung hineingewachsen. Mit der Zeit haben sehr viele inzwischen etablierte neue Politiker nicht nur die Positionen ihrer früheren Peiniger übernommen, sondern teilweise auch deren Ansichten, Lebensweisen ${ }^{484}$ und Vorlieben für bürokratische Aufblähungen des „Verwaltungsapparates ${ }^{4485}$.

Ziemlich pointiert beschreiben Adam, van Zyl Slabbert und Moodley am Ende ihres Werkes über ehemalige Genossen, die jetzt „,im Business“ tätig seien, die

481 Business Day, 11.11.1997: „Bank Plans Set of Reforms for Markets“.

482 Ohne Verfasser (1997b). Später wurde das MTEF noch durch eine MTBPS (Medium Term Budget Policy Statement ersetzt (vgl. Mail \& Guardian vom 14.11.2003: „A calculated gamble").

483 Vgl. Handelsblatt, 05.01.1998: „Der Himmel über der Kaprepublik hat sich etwas aufgehellt".

484 Vgl. hierzu Adam/van Zyl Slabbert/Moodley (1998), insbesondere S. 160ff.

485 In einer internen Mitteilung des Außenministeriums an die Botschaften Südafrikas wird unter der Überschrift „More Staff for Mbeki“ gemeldet, dass die Anzahl der Mitarbeiter im Büro des Vizepräsidenten sich in nur drei Jahren weit mehr als verzehnfacht hatte („officials employed by Deputy President Thabo Mbeki's Office increased from nine in September 1994 to 121 in September 1997." Und zwar noch im vorletzten Jahr seiner Präsidentschaft (1998), wo Präsident Mandela sich schon als „semi retired“ (Johnson (2009), S. 139) betrachtete und seinem für die Nachfolge vorgesehenen Vizepräsidenten immer mehr administrative Aufgaben abzutreten begann, so dass Mbeki schon als „de facto prime minister" (Gevisser (2013), S. 658) unter dem Präsidenten Mandela, ziemlich, aber nicht völlig frei fungieren konnte (vgl. auch Gevisser (2010)). 
soziale und ökonomische Situation zum Ende des ersten Übergangsjahrzehnts. Nach nur fünf Jahren demokratischer Regierung des ANC, wie die Verfasser im Abschlusskapitel ihres Buches „Comrades in Business": „The underclass versus the liberation aristocracy ${ }^{\text {c486 }}$ beklagen.

Während einige wenige aus der Armut aufgestiegen seien ${ }^{487}$, blieb in den etwa fünf bis zehn Jahren die Mehrheit arm und wurde noch ärmer, stellen z. B. Adam, van Zyl Slabbert \& Moodley und Bond am Ende mit sichtlicher Enttäuschung fest. Und auch ein nordamerikanischer Gast aus Kanada bzw. ein, eine Zeit lang, wie es Soziologen oder Psychologen wohl ausdrücken würden, „,beobachtender Teilnehmer“ bzw. „outsider“ Südafrikas, der Wissenschaftler David M. Mathsinshe ${ }^{488}$, beschrieb

486 Adam/van Zyl Slabbert/Moodley (1998), S. 201 bzw. S. 201-225.

487 Solche sozial aufgestiegenen Menschen einer seit etwa zehn Jahren entstehenden „black (upper) middle class", wie es in südafrikanischen Medien allenthalben zu lesen steht, wurden teils in allem Ernst und mit einer gewissen Anerkennung „black diamonds“ genannt, teils aber auch gehässig, besonders für Unternehmer („entrepreneurs“), welche ihren neu erlangten Reichtum und Einfluss nutzten, um mit ihren auch erlangten "political connections" (zum ANC) Verträge zu erhalten, die ihnen sonst verwehrt geblieben wären („to obtain contracts they would otherwise be denied“ (Maja (2017), S. 1),. Der Begriff „black diamonds" ist auch im US-amerikanischen, teils auch im britisch-englischen Sprachgebrauch üblich. Im Englischen würde man eher den Begriff, ,fat cats" verwenden. Black Diamonds gehört zur Umgangssprache Südafrikas, wird in den Massenmedien (vgl. z. B. McLean 2008) verwendet und steht auch in wissenschaftlichen Publikationen - teils kritisch verwendet bzw. kritisiert (vgl. z. B. den Soziologen Southall (Southall (2016)) oder de Coninck (2018), teils, z. B. von südafrikanischen, israelischen bzw. israelisch-englischen und schwedischen Wissenschaftlern offenbar völlig unkritisch verwendet (vgl. z. B. Kitis, Milani \& Levon (2018)) zu lesen. Seit seiner Prägung durch einen BWL- bzw. Marketing-Professor der Kapstädter Universität (UCT), Prof. John Simpsons, landesweit bekannt gemacht durch Veröffentlichung einer Studie im Business Club in Johannesburg im Juni 2008, in der von einer sich entwickelnden „black middle class"mit den „black diamonds", geschrieben wird (vgl. auch Mail \& Guardian vom 22.07. 2007: „Study shows SA black middle class booming“, und vom 31.01.2014: „SA's mysterious 'middle class" und MacLean (2008)), ist der Begriff „black diamonds" zu einem in Südafrika in Gesprächen oder in Telephongesprächen mit in Südafrika wohnenden Menschen täglich zu hörenden umgangssprachlichen Begriff geworden. Wie Mpofu und Gatshani-Ndlovu in einem Fachaufsatz im Journal of Critical African Studies andeuten bzw. sogar am Beispiel des inzwischen verstorbenen Staatspräsidenten Mandela beschreiben (vgl. Mpofu 2018 \& Gatshani-Ndlovu (2018a)), haben junge wie ältere Südafrikaner bis heute damit Probleme, den auch gedanklich bzw. bis in die Sprachgewohnheiten bzw. den Sprachgebrauch hineinreichenden Wandlungsprozess weg von einer früheren Schwarz-Weiß-Dichotomie vorzunehmen und zwar „as an essial pre-requisite for the rebirth of a new political community" (Mpofu 2018b) in Südafrika.

488 Vgl. Adam, van Zyl Slabbert \& Moodley (1998), Bond (2003), Bond (2007), Bond (2010a) und Bond (2014a)), und auch Matsinhe, der sich selbst, zur Verstärkung und Fundierung 
die sozioökonomische Situation Südafrikas (immerhin im sechsten „Dienstjahr“ einer de facto ANC-Alleinregierung seit dem Frühling 1994) ähnlich detailliert und mit unrühmlichen Beispielen ähnlicher Art.

Selbst Jahre später, nach dem von der „Parteilinken“ des ANC erzwungenen Rücktritt Mbekis (Ende 2008) ${ }^{489}$ und der Vereidigung Präsident Zumas (Mai 2009), wunderten sich Adam \& Moodley immer noch über die Metamorphose so vieler ehemaliger armer, zu Unternehmern bzw. Geschäftsleuten gewordener Genossen („Comrades in Business"). Vor allem über die Geschwindigkeit, mit der ehemalige, einst durch und durch überzeugte Kommunisten sich in „Erzkapitalisten“ gewandelt hatten ("formerly staunch communists hat turned into arch-capitalists ${ }^{(490}$ ). Beide Wissenschaftler fragten sich, wann bzw. ob es der so zugängliche und freundliche („affable") neue Präsident Zuma, unter Anweisung seiner Berater, schaffen würde, die Anglo American Corporation ${ }^{491}$ zu verstaatlichen. ${ }^{492}$

Tatsächlich hat sich in den letzten etwa zehn Jahren eine - noch kleine - schwarze (eher obere) Mittelschicht ${ }^{493}$ gebildet, die sich auch als „schwarz“ empfindet, sich

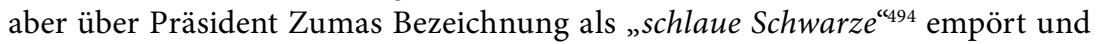
zu der z.B. Ärzte, Architekten und Ingenieure, Ökonomen, Wirtschaftsprüfer,

seiner eigenen Beobachtungen und Aussagen, auch abschließend teilweise auf die schon zitierte Literatur bezieht.

489 Kommentatoren sprachen auch von „Putsch“ oder „Partei-Putsch“, vgl. z. B. Weber (2008)). Danach erhielt Südafrika bis zum Ende der regulären Amtszeit Mbekis den bloßen Übergangspräsidenten Kgalema P. Motlanthe, der letztlich nur für eine geordnete und „gesichtswahrende“ Übergabe von Präsident Mbeki an Präsident Zuma sorgte und dafür noch bis 2014 als Zumas Vizepräsident fungieren durfte.

490 Adam \& Moodley (2010), S. 52.

491 Seit 1999: Anglo American plc.

492 Adam \& Moodley (2010), S. 52, S. 53.

493 Der Begriff „Mittelschicht“ wird hier als Übersetzung dessen verwendet, was in der englischsprachigen wissenschaftlichen Literatur zumeist mit „middle class“ bezeichnet wird, nicht in einem marxistischen Sinne, zumal Marx selbst keine vollständige Analysen von „Klassen“" geboten hat bzw. keine mehr bieten konnte, weil er den dritten Band des Kapitals abbrach, als er sich damit beschäftigt hatte, was Klassen seien (vgl. Thompson 2001, S. 65). Es geht hier nur um eine demographisch-soziologische Sichtweise einer in sozialen Schichten entwickelten Bevölkerung, um die Bezeichnung jener Schicht, die nach Kultur, Bildung und Ausbildung, Einkommens- und Vermögensausstattung etwa in der Mitte einer nach sozialen Schichten strukturierten Gesellschaft, d. h. zwischen einer Ober- und Unterschicht rangiert und selbst wieder in eine obere, mittlere und untere Mittelschicht eingeteilt wird, ohne diese Bevölkerungsgruppe, wie in allen Ländern, wirklich ganz exakt und ohne Widersprüche abgrenzen zu können.

494 Ngoma (2014), S. 72. 
höhere Beamte und hoch qualifizierte Fachkräfte im Finanzdienstleistungssektor der Banken und Versicherungen gehören ${ }^{495}$.

Nicht nur in Südafrika, sondern weltweit scheinen Politiker sehr oft die wichtige, demokratiestabilisierende Funktion der Mittelschicht eines Landes, deren weiteres Wachstum gerade in Südafrika mit seinen besonders großen Unterschieden zwischen Arm und Reich wichtig wäre, zu unterschätzen, zumal rund zwei Drittel der Bevölkerung Südafrikas immer noch als „arm“ einzustufen sind ${ }^{496}$, ohne an dieser Stelle eine „exakte“ oder „richtige“ Armutsschwelle definieren zu müssen ${ }^{497}$.

In Zeiten, in denen eben nicht nur in Südafrika sondern auch weltweit eine zunehmende soziale Ungleichheit bzw. deren Bekämpfung zum Beispiel von der Präsidentin des Internationalen Währungsfonds, Christine Lagarde, oder von Präsident a.D. Barak H. Obama zu einer epochalen Aufgabe erklärt wurden ${ }^{498}$, wird der Wert einer gesellschaftliche Spannungen abfedernden und dabei gleichzeitig die Gesellschaft zusammenhaltenden Mittelschicht, und zwar auch in ihrer Vorbildfunktion und durch Förderung typischer Werte der Angehörigen einer Mittelschicht bzw. Tugenden wie Fleiß, Disziplin und Sparsamkeit, die auch die soziale Mobilität im Sinne einer Aufwärtsmobilität fördern, leider zunehmend unterschätzt ${ }^{499}$. Gerade Angehörige der Mittelschicht und die soziale Mobilität der Aufsteiger aus der oberen Unterschicht leisten überdurchschnittlich viel für den Zusammenhalt und die Finanzierung demokratischer Gesellschaften, denn, wie z. B. auch in Deutschland, zahlen sie die meisten Steuern ${ }^{500}$ und Sozialbeiträge, insbesondere Einkommensteuern und Kranken-, Arbeitslosen- und Rentenversicherungsbeiträge.

495 Vgl. Ngoma (2014), S.62-72.

496 Vgl. Bauer \& Taylor (2011), S. 295.

497 Solcher Armutsschwellen („poverty lines“) gibt es mehrere und alle sind umstritten, im Abschnitt 3.1.1 wurde die Thematik bereits angeschnitten. Auch in der Bundesrepublik Deutschland ist man sich weder unter Politikern noch unter Ökonomen darüber einig, wie die Menge der „Armen“ einzugrenzen ist bzw. wer tatsächlich „arm“ ist, zumal der Begriff selbst in der heutigen Bundesrepublik Deutschland eher als „oberflächlich“ oder sogar politisch nicht korrekt eingestuft wird.

498 Vgl. Atkinson (2017), S.7.

499 Vgl. Sprenger-Menzel (2018), S. 334-335.

500 So wurde und wird, auch in der Bundesrepublik Deutschland, trotz hoher Spitzensätze für hohe Einkommen, mehr als die Hälfte der Einkommensteuer nicht von den „Reichen“ der Gesellschaft, sondern von Menschen erbracht, die weniger als 6.000 Euro im Monat bzw. weniger als 80.000 Euro im Jahr verdienen (vgl. auch „Das Märchen von den Reichen“, in: Die Zeit v. 8. 12.2011). 
Deshalb kommt - wie in jedem Land der Erde - auch in Südafrika eine starke und wachsende Mittelschicht üblicherweise der gesamten Gesellschaft und besonders der Unterschicht und den Armen zugute, weil deren Beitrag zu den Sozialversicherungssystemen bzw. zur Haushaltsfinanzierung des Staates überproportional hoch ist. Den Angehörigen der aus der Unterschicht aufgestiegenen Angehörigen der neuen schwarzen Mittelschicht in Südafrika sollte ihre mögliche, ja sogar gebotenen Vorbild- und Motivationsfunktion für die Armen Südafrikas bewusster sein.

Üblicherweise engagieren sich Angehörige der Mittelschicht in besonders intensiver Weise in ehrenamtlichen Organisationen und tragen besonders häufig und intensiv zu kulturellen, politischen und sozialen gesellschaftsstabilisierenden Prozessen bei ${ }^{501}$. In Südafrika könnte und $\mathrm{m}$ u s s die Mittelschicht weiter wachsen - und $\mathrm{m}$ it der Mittelschicht Südafrikas gerade auch die sozial benachteiligten Schichten, was am Ende dem ganze Lande zugute käme, während in vielen Industrieländern der nördlichen Halbkugel die Mittelschicht stark unter finanziellem Druck steht und anscheinend nicht nur quantitativ kleiner sondern auch in ihrer gesellschaftsprägenden und gesellschaftsstabilisierenden Funktion kleiner zu werden scheint, und zwar inzwischen derart deutlich, dass Regierungen und Politiker in den letzten Jahren die Mittelschicht „wiederentdeckt" haben und diese finanziell, d.h. steuerlich stärker entlasten wollen, wie z. B. in Nordamerika, Österreich oder besonders in England ${ }^{502}$.

Doch diese schwarze Mittelschicht ist in die feineren Vororte (suburbs) gezogen, beschäftigt private Sicherheitsfirmen ${ }^{503}$, weil ihr die Polizei kein Sicherheitsgefühl vermittelt, geht nur noch zur Polizei, um Fallnummern (für die Begleichung von Versicherungsschäden etc.) zu erhalten, und scheint, jedenfalls selbst noch am Ende der Ära Mbeki, von den sozialen „Basisinstitutionen“ der südafrikanischen Gesellschaft, wie z. B. Schulen, Krankenhäusern und Polizei, „meilenweit entfernt ${ }^{\text {“504. }}$.

Für Südafrika am Ende der 1990er Jahre, bei allen Schwierigkeiten des Landes, z. B. mit der weiterhin weitaus zu hohen sozialen Ungleichheit und der hohen und weiter zunehmenden Kriminalität ${ }^{505}$, lässt sich das Zwischenfazit ziehen, dass sich

501 Vgl. auch Gelb (2008), S. 90.

502 Vgl. Times v. 18.03.2016: „Squeezed middle gets helping hand“ und „Chancellor makes saving interesting for the under-40s“, in: Times v. 19.03.2016.

503 Vgl. z. B. die Fidelity ADT, eine waffentechnisch „gut ausgerüstete“ Sicherheitsgesellschaft mit Sitz in Midrand, $30 \mathrm{~km}$ südlich von Pretoria, die BBBEE-akkreditiert ist und in Südafrika und weiteren 35 Ländern Sicherheits- und Brandschutzdienste anbietet (vgl. für ein Unternehmensportrait Fidelity ADT (2019)).

504 Wilke-Launer (2010), S. 224.

505 Hierüber schien sich der Südafrikakenner Prof. Heribert Adam (Vancouver University, Kanada, und mehrmals Gastprofessor (Visiting Professor) an Südafrikanischen 
die Demokratie in Südafrika auf jeden Fall stabilisiert hat, doch darf man mit Adam/ van Zyl Slabbert/ Moodley (1998) auch konstatieren, dass ,the real test for the future of South African democracy is whether it is based on an inclusive majoritarianism or ethnic majoritarianism ${ }^{\text {"506 }}$. Hier gibt es, so scheinen es auch die sozioökonomischen Realitäten im Jahre 2019 anzuzeigen, für die Regierung und die gesamte Gesellschaft in der Rainbow Nation des Neuen Südafrikas immer noch viel zu tun.

\subsection{Wendungen nach außen (African Renaissance und NEPAD) und Rückwendungen nach innen (Affirmative Action und BEE)}

Rund ein halbes Jahrhundert nachdem die ersten Kolonien in Asien (1947-1948 die heutigen Nationen Bangladesch, Indien, Myanmar, Pakistan und Sri Lanka) und (ab 1951 beginnend mit Libyen, dann v.a. Mitte der 1950er Jahre bis Mitte der 1960er Jahre) die meisten Länder Afrikas in die Unabhängigkeit entlassen worden waren und nun auf den Jahrtausendwechsel zugingen, war eine gewisse Trägheit und Ineffektivität überstaatlicher, gesamtafrikanischer Organisationen, wie z. B. der von 1963-2002 bestehenden Organization of African Unity (OAU mit 53 Mitgliedern, seit 2002 African Union (AU) mit derzeit 55 Mitgliedsstaaten), zu

Universitäten, z. B. an der UCT) schon 1995 sehr zu wundern, besonders darüber, dass nach der erstaunlichen demokratischen Transformation zwar die politisch motivierten Morde zurückgingen, aber die kriminelle Gewalt schon Mitte der 1990er Jahre, nach Antritt der ANC-Regierung derart stark angestiegen war (vgl. Adam (1995)). Gerade in den Städten Südafrikas, wie in vielen anderen zentral-, west- und südafrikanischen Länden, war die „urban violence“ derart stark, dass wissenschaftliche Literatur mit Titeln wie „Städte unter Belagerung“ mit einer Reihe von Aufsätzen zur urbanen Gewalt und Kriminalität erschienen (vgl. z.B. Glanz (1995), den Überblick unter dem Titel „Cities under Siege“ mit Beiträgen zur „Urban Violence in South, Central and West Africa" von Louw \& Bekker (1996)). Bis Ende der 1990er Jahre war die Kriminalität derart umfassend und weiter im Steigen begriffen, dass sogar Präsident a.D. Mandela glaubte, sich darüber beschweren zu müssen, dass „zu viel“ Berichterstatttung über die grassierende Kriminalität Südafrikas zu einem Wegbleiben oder sogar zu einer Flucht der Investoren geführt hätte und die Presse sich mit der Berichterstattung über Kriminalität „unpatriotisch“ “verhalte (vgl. Gumede \& Chauke (2003), S. 99). Doch auch noch viele Jahre später bei Russel (2010), S. 107-132 findet sich ein Kapitel mit dem klaren Titel "City under Siege“ und die Aussage "South Africa's crime rates are extraordinary“ (ebd., S. 109). Ähnliches wird auch für das zweite Halbjahr 2018 aus südafrikanischen Städten von Feltes berichtet (vgl. Feltes (2018), passim).

506 Ebd. bzw. Adam/van Zyl Slabbert/Moodley (1998), S. 158. 
erkennen. Nicht mehr alle (damals) jungen Staatsführer Afrikas wollten dies weiter akzeptieren. Auch mit dem Wunsch nach mehr politischer und ökonomischer Unabhängigkeit vom „westlichen“ Ausland und internationalen Finanzquellen und nach der Nutzung eigener Rohstoffe und Ressourcen, gewiss aber auch wegen des in Staaten Afrikas wieder wachsenden Bewusstseins, dass im Interesse der Wahrung der Zukunftsfähigkeit ihrer Länder nicht ohne Not alle afrikanischen Werte und Traditionen aufgegeben bzw. in Vergessenheit geraten sollten, wandten sich z.B. der ugandische Präsident Yoveri Kaguta Museveni, der ein „afrikanisches Erwachen“ und Präsident Thabo M. Mbeki, der sogar eine „afrikanische Wiedergeburt“ („African Renaissance“) spürte und hierbei für Südafrika eine Art Schlüsselrolle anstrebte $^{507}$, an andere Staaten Afrikas, um frühere Fehlentwicklungen, die teils noch mit der Dekolonisation zu tun hatten, hinter sich zu lassen. ${ }^{508}$

Mit der neuen ökonomischen Partnerschaft für afrikanische Entwicklung (New Partnership for Africa's Econonomic Development, NEPAD) - auch als integraler Bestandteil der African Union (AU) ${ }^{509}$ - sollte gegen den „Afrikapessimismus“ angekämpft, ein Bekenntnis zur Eigenverantwortung der Staats- und Regierungschefs Afrikas abgegeben ${ }^{510}$ und als ein lockerer Bund zur Förderung der regionalen Entwicklung der Staaten Afrikas gegründet werden, um damit Investitionen und die Schaffung von Arbeitsplätzen zu fördern. Damit hoffte man auf das Eintreten von Verstärkungs- bzw. Multiplikatoreffekten vom Kap bis nach Kairo. Hierzu setzten sich die Staaten Afrikas hohe Ziele (wie viele von Anfang an kritisierten, zu hohe Ziele), ohne gleichzeitig klare Zeitrahmen für deren Erfüllung vorzugeben, doch Präsident Mbeki betonte ständig, dass die Ziele „ehrgeizig“ („ambitious“) sein müssten, um damit auch eine „moralische Verpflichtung“ („moral obligation“) zum Vorantreiben der Ideen und zur Förderung der Entwicklung Afrikas zu schaffen. ${ }^{511}$

$\mathrm{Zu}$ den wichtigsten Ziel der NEPAD-Initiative, die jedem, der mit dem Inhalt und den Zielen der südafrikanischen Programme RDP, GEAR und AsgiSA (Accelerated

507 Worden (2008), S. 165.

508 Vgl. auch Schlicht (1998), S. 70.

509 Vgl. Venter (2003), S. 254. Einen guten Gesamtüberblick zu NEPAD bieten Bobo \& Sintim-Aboagye (2012), passim.

510 Vgl. Jakobeit (2006), S. 21.

511 Vgl. Venter (2003), S. 240 (dort auch die in englischer Sprache zitierten Einschätzungen Mbekis). 
and Shared Growth Initiative for South Africa, das 2006 startete $^{512}$ ) vertraut ${ }^{513}$ ist, teilweise (sehr) bekannt vorkommen dürften, gehörten:

- die Schaffung von Frieden und Sicherheit in (ganz) Afrika,

- die Vernichtung von Armut, Unterentwicklung und Einkommens- und Vermögensungleichheiten zwischen „Arm“ und „Reich“,

- die Förderung beschleunigten Wachstums und nachhaltiger Entwicklung,

- die Beendigung der Randstellung Afrikas im Prozess der Globalisierung, wo die Staaten Afrikas weniger als 2 Prozent des Welthandels (1,7\%), 2 Prozent der Exporte der Welt und 0,9 Prozent der globalen ausländischen Direktinvestitionen (Foreign Direct Investments, FDI) erhielten,

- der Ausbau und eine bessere Finanzierung des Sozialstaates und

- die Verbesserung der Gesundheitsvorsorge und Seuchenprävention (vor allem von HIV/AIDS, Malaria und Tuberkulose). ${ }^{514}$

Am Ende der mehrjährigen Bemühungen zeigte sich, dass sowohl die Initiative NEPAD als auch die besonders von Präsident Mbeki propagierte Kampagne einer „African Renaissance“, zu deren Verbreitung es sogar Veranstaltungen in Europa, z. B. in Bonn, gab, ${ }^{515}$, so altruistisch und alle Seiten gegenseitig fördernd sie auch wirken sollten, in der Hauptsache zu einem politischen Imagegewinn des ANC sowie zu hohen Gewinnen vor allem südafrikanischer Unternehmer und Privatleute geführt hatte..$^{516}$

Der Erfolg der NEPAD-Partnerschaft ${ }^{517}$ litt am Ende auch darunter, dass das fast allumfassende Mandat (Außenhandel, Schuldenerleichterungen, Entwicklungshilfe-Koordination, Infrastruktur-Investitionsmaßnahmen, ausländische

512 Vgl. weiter unten im Abschnitt 5.3.1.

513 Was selbstverständlich auch daran liegt, dass die Entwicklungsprobleme afrikanischer Staaten, z. B. in der Armutsbekämpfung, der Landwirtschaft und Ernährung, der Bildungsungspolitik, des öffentlichen Dienstes etc. überall in Afrika auf „Roadmaps to Africa's Renewal“ ((Cheru (2008), S. 36) zu lesen stehen (vgl. ebd. und auch S. 37-38).

514 Vgl. Cliffe (2002), Prah (2002) und Venter (2003), S. 239-246.

515 Vgl. detailliert hierzu Hunter-Gault (2008). Sehr viel Schwung wurde der Renaissance-Kampagne auch tatsächlich durch die HIV/AIDS-Pandemie genommen (vgl. z. B. Shell, Quattek, Schönteich \& Mills (2000)).

516 Vgl. Schröder (2012), S. 5.

517 Für eine weitere wissenschaftliche Diskussion pro und contra NEPAD vgl. z. B. Games (2004), Grobbelaar (2004), etwas kritischer Bond (2002) und auch Alden \& Soko (2005) und Samson (2009) sowie, für einen die Diskussion zusammenfassenden Überblick Miller, Oloyede \& Saunders (2008). 
Direkt-Investitionen (FDI), innerafrikanische Konfliktlösungen etc.) doch eher diffus ${ }^{518}$ war. Die wenigen präziser formulierten (und damit operationalisierten) NEPAD-Ziele, d.h. die verabredeten sozioökonomischen Ziele, die teils durchaus mit den Grundzielen des RDP konform gingen, haben die meisten afrikanischen Länder leider nicht erreichen können. Zum Ende der Ära Mbeki hatten es gemäß der Zielsetzungen des New Partnership for Africa's Development (scorecard of the NEPAD) nur sieben von 53 Ländern Afrikas (Äthiopien, Burkina Faso, Kap Verde, Malawi, Mali, Niger und der Tschad) tatsächlich, wie verabredet, erreicht, zum Abbau der Armut und des Hungers in Afrika - wenigstens - zwischen fünf und zehn Prozent für landwirtschaftliche Zwecke auszugeben.

Alle Länder scheinen darunter zu leiden, dass die zu bebauenden Flächen zu klein sind, um sie wirtschaftlich zu bearbeiten, zumal das Privateigentum an Grundstücken bzw. landwirtschaftlicher Nutzfläche oft ein Problem ist. Das Land, das in vielen Ländern Afrikas entweder von der Regierung oder von einem lokalen ,traditional leader" oder „tribal chief " “519 vergeben wird, weist häufig für eine wirtschaftliche und dabei auch technisch moderne landwirtschaftlichenNutzung eine zu kleine Fläche auf. Hier spielt auch die ordnungspolitisch wie „psychologisch“ wichtige Frage eine Rolle, ob Land tatsächlich in das Eigentum der Landwirte übergeht, oder ob sie nur für eine begrenzte Zeit lang Pächter sein dürfen.

Die Landflucht in afrikanischen Ländern, auch Teilen Südafrikas, könnte verhindert werden, wenn es klare und auch sichere Eigentumsverhältnisse oder z. B. wenigstens „Erbpachten“ über 99 oder mehr Jahre geben könnte, um den Landwirten das Vertrauen zu vermitteln, dass sich langfristige Investitionen, z. B. in Bewässerungssysteme, lohnen bzw. sich vielleicht wenigstens in den Folgegenerationen auszahlen. ${ }^{520}$

518 Vgl. auch Butler (2009), s. 64-65.

519 Thurow \& Kilman (2009), S. 268.

520 Vgl. Thurow and Kilman, S. 269. Ein großes, zur Zeit oft das größte Problem ist, dass selbst in den drei wohl fruchtbarsten und dabei auch großflächigen Ländern Afrikas, nämlich im Kongo, Simbabwe und Sudan, wegen Kriegen und Bürgerkriegen die Landwirtschaft brachliegt. Hier wie früher einst in Simbabwe, könnte der Brotkorb für das ganze südliche Afrika und wohl weitere große Teile Afrikas liegen, der seit Jahrzehnten nicht mehr genutzt wird, weil es lebensgefährlich geworden ist, dort Landwirtschaft zu betreiben (vgl. ebd.), denn seit Jahren ist von dort nur von Krieg und Bürgerkriegen, von Völkermorden, Kindersoldaten, Seuchen etc. zu hören, so dass man auf der nördlichen Halbkugel und im sogenannten „Westen“ bei einem oberflächlichen Blick auf Afrika zwischen zwei Extrema hin- und hergerissen wird. Easterly formuliert hierzu: „IN THE WEST, AFRICA'S IMAGE SEEMS FOREVER torn between two extremes: Politically Correct Positive and Stereotypically Negative."Aber dass sogar dann wenn der Fortschritt zerbrechlich (zart) gilt:"the case for hope in Africa is on the most solid of 
Anderes wichtigee Gebiet waren Präsident Mbeki wie dem ganzen ANC die Herstellung von Chancengleichheiten durch Maßnahmen einer Affirmative Action und ein gezieltes Black Economic Empowerment.

Schon im Reconstruction and Development Programme werden die Begriffe "Affirmative Action" und „Black Economic Empowerment" bei der Vorstellung der RDP-Ziele bzw. Handlungsfelder "Developing our Human Resources“, „Meeting the Basic Needs", „Social Security and Social Welfare“, „Higher Education“ "Labour and Worker Rights", „National and Provincial Government", ,Public Service“, „Democratic Information Programme“, ,Restructuring the National Budget", und "The Corporate Sector" insgesamt zwanzig Male ausdrücklich verwendet.

In enger Anlehnung an die während der nordamerikanischen anti-rassistischen Bürgerrechtsbewegung ${ }^{521}$ zur Erreichung der Gleichberechtigung und Gleichstellung afroamerikanischer Mitbürger entwickelten wirtschafts- und gesellschaftspolitischen Maßnahmen einer Politik der Affirmative Action (v. a. im gesamten Bildungswesen, im Gesundheitswesen und im Arbeitsleben), mit dem Ziele bewusster und gezielter Vorteilsgewährung durch besondere Unterstützung, Bestätigung und Vorteilsgewährung bei der Einstellung, Ausbildung, Fortbildung und Beförderung schwarzer Südafrikaner im Berufsleben, sieht auch das RDP die Politik der Affirmative Action (AA) und des Black Economic Empowerment (BEE) ausdrücklich als Instrumente bzw. als geeignete, ja gebotene Maßnahmen der sozioökonomischen Transformation Südafrikas. Im Jahresbericht der BEE Commission 2001 formulierte diese im Prolog daher konsequent:

"The BEECom believes that an Intergrated (sic!) National BEE Strategy is intergral (sic!) to the success of the RDP. At the same time it will launch South Africa on to a course of sustained and even spectacular rates of economic growth." 522

Die BEE Commission sieht das gesamte Projekt des Black Economic Empowerment als einen integrierten und zusammenhängenden sozioökonomischen Prozess: „It is located within the context of the country's national transformation programme, namely the RDP."

possible foundations: the resourcefulness and creativity of the African people themselves" (Groß- und Kleinschreibung aus der zitierten Quelle übernommen).

521 Das bis an den Anfang des XX. Jahrhunderts zurückreichende Civil Rights Movement in den USA mit einem Mitte der 1950er bis Ende der 1960er Jahre erreichtem Höhepunkt unter dem nordamerikanischen Bürgerrechtler Martin Luther King.

522 BEECom (2001), S. V. Die Orthographiefehler wurden vom Verfasser nicht korrigiert; der Text wurde unverändert aus dem vom damaligen Chairman und 2019 wiedergewählten Staatspräsidenten Cyril Ramaphosa herausgegebenen Report zitiert. 
Trotz nationaler wie internationaler Kritik, weil Affirmative Action ja selbst schon wieder eine neue erniedrigende und Lebenschancen vorenthaltende - und im Einzelfalle sogar objektiv ungerechte - Diskriminierung zu Gunsten früher selbst diskriminierter Teile der Bevölkerung bedeute ${ }^{523}$, führte Südafrika unter der Regierung des ANC seit 1994 - nach mehr als vier Jahrzehnten (gerechnet seit mindestens 1948) rassischer bzw. rassistischer Diskriminierung im Verlauf der Apartheid-Ära - eines der wohl weitreichendsten und ehrgeizigsten Affirmative Action-Programme der ganzen Welt ein. ${ }^{524}$ Hierbei ging es im RDP nicht nur um berufliche und damit auch wirtschaftliche und gesellschaftliche Doppelbenachteiligungen, also nicht nur um die allgemeine ökonomische Ertüchtigung und Besserstellung, „Black Economic Empowerment" (BEE), auch „upliftment" genannt) sondern vielmehr auch um ein empowerment junger schwarzer Mädchen und Frauen. ${ }^{525}$

Als „positive“ Diskriminierung im Sinne der Affirmative Action sollte das südafrikanische Black Economic Empowerment (BEE) den bisher benachteiligten Bevölkerungsgruppen im Sinne der Verfasser des RDP schon beim Zugang zur Berufsausbildung, während der Ausbildung und des ganzen Berufslebens auf dem ganzen Arbeitsmarkt bessere Chancen erwirken. Im Prinzip geht es auch darum, dass sich die sozio-demographische Struktur der südafrikanischen Bevölkerung auch in der Struktur der Beschäftigten der Unternehmen widerspiegelt. Hierfür sollte der Employment Equity Act durch Förderung der Chancengleichheit sorgen. Auch für eine faire Behandlung durch die Vermeidung unfairer Diskriminierung und die Anwendung von "Affirmative Action Measures ${ }^{4526}$, für eine faire Präsenz in allen beruflichen Kategorien und hierarchischen Ebenen der Mitarbeiter. ${ }^{527}$ Dies scheint wohl nicht in der Breite der Unternehmen und v. a. nicht bei den kleineren und mittleren Unternehmen zu funktionieren, aber bei den Großunternehmen, insbesondere in der Industrie, offenbar schon. ${ }^{528}$

523 Vgl. z. B. Shubane (1995), S.2-24, der die Leistungsgerechtigkeit betont, welche durch Affirmative Action untergraben werden könne.

524 Vgl. hierzu die Einleitung bei Jeffery (2014), S. 1.

525 „Women must be trained to be scientists and doctors, not only nurses and teachers" (Biehl (1994), S 102.

526 Wörtlich zitiert aus dem Chapter I (Definitions, Purpose, Interpretation and Application), Purpose of this Act, $2 a$ and $2 b$. (ILO (o. J.). Zu betonen wäre noch, dass in dem Gesetz noch definiert wird: "Black people" is a generic term which means Africans, Coloureds and Indians"(ebd.).

527 Vgl. ebd. und vgl. auch South African Labour Guide (2019), S. 1.

528 Vgl. hierzu diverse Beispiele zur Affirmative Action und zum BEE aus der Industrie, z. B. in der Automobilindustrie (BMW) in Südafrika, wo es viele Versuche einer Umsetzung der Anforderungen des BBBEE gibt, aber der Verfasser einer Dissertation zum Thema 
Zwar wurde auch ein BEE Advisory Council gegründet und 2005 wurden Durchführungsrichtlinien (Codes of Good Practice) eingeführt, doch offenbar konnten in der Praxis nur wenige Arbeitnehmer davon profitieren. Hier sollten dann weitere Modifikationen in den nächsten Jahren und ein neues Gesetz helfen; der Broad-based Black Economic Empowerment Amendment Act. Die Änderungen traten 2014 in Kraft. ${ }^{229}$

Bei der Umsetzung des BEE zeigten sich in der Praxis Schwierigkeiten in Schulen, Unternehmen und in der öffentlichen Verwaltung, vor allem die Schwierigkeit, dass das BEE zwar einigen schwarzen Investoren und (ANC-) Politikern die Möglichkeit verschaffte, davon zu profitieren, die breite Masse, also die „Basis“ der schwarzen Bevölkerung Südafrikas aber nicht erreichte. Deshalb verabschiedete die Regierung zur Erreichung einer breitenfördernden bzw. breit angelegten Umsetzung des BEE einen „Broad Based Black Economic Empowerment Act (BBBEE). Hierzu gehörte u. a. ein komplexes, auf Basis eines Punktesystems („BEE Scorecard System“) und mit der Vergabe von „BBBEE-Gütesiegeln“ aufgebautes Verfahren, für welches wiederum eigens Akkreditierungsagenturen errichtet wurden, mit denen die Unternehmen dann hauptsächlich mit Online-Formularen kommunizieren. Die Akkreditierungsagenturen sollen auch die Aufsicht über die Einhaltung der Ziele des BBBEE durch die Unternehmen führen.

Unternehmen, die öffentliche Aufträge in Südafrika erhalten wollen, müssen möglichst schwarze (Mit-) Inhaber vorweisen, nachweislich Mitarbeiter beschäftigen, die einer farbigen Minderheit (z. B. Asiaten, Inder und Farbige) angehören und auch „beweisen“, dass alle Angehörigen des Unternehmens gleiche Rechte und Chancen haben. Hierzu wurde ein inzwischen mehrfach geändertes und immer wieder weiter optimiertes Bewertungssystem eingeführt, mit dem das DTI (Handels- und Industrieministerium Südafrikas) in Kategorien einer Gesamtrechnung (Scoringtabelle bzw. der Punktwerttabelle) zu einer BBBEE-Zertifizierung zusammengefasst werden.

$\mathrm{Zu}$ den Bewertungskategorien, die dann jeweils noch gewichtet werden, gehören die Eigentumsverhältnisse, die Mitbestimmung bzw. Managementkontrolle, die Beschäftigungsgleichheit, die Entwicklung von Kompetenzen (Fortbildung), Beschaffungspräferenzen, die Unternehmensentwicklung und eine sozioökonomische Entwicklung.

„Affirmative Action“ und „BBBEE“ am Ende konstatieren muss, dass seine empirischen Erhebungen offenbart haben, dass sie (jetzt) noch nicht sinnvoll seien und die Industrie die (An-) Forderungen des BBE (noch) nicht erfüllen könne und es zu früh sei, die Wirkungen der Affirmative Action und des BEE zu messen (vgl. Hecht, S. 212-246).

Vgl. den offiziellen Text in DTI (2014). 
Tab. 10 Einfaches Beispiel für ein BBBEE-Scoring (BBBEE Scoringcard) ) $^{530}$

\begin{tabular}{lc}
\hline Bewertungskategorie & Gewichtung \\
\hline Eigentumsverhältnisse & $20 \%$ \\
\hline Mitbestimmung/Managementkontrolle & $10 \%$ \\
\hline Gleichheit der Mitarbeiter & $15 \%$ \\
\hline HR-Entwicklung (Aus- und Fortbildung) 0 & $15 \%$ \\
\hline Beschaffungspräferenz & $20 \%$ \\
\hline Unternehmensentwicklung & $15 \%$ \\
\hline sozioökonomische Entwicklung & $5 \%$ \\
\hline
\end{tabular}

$\mathrm{Ab}$ einem Jahresumsatz von 5 Millionen Rand ${ }^{531}$ müssen sich auch schon kleinere Betriebe mit vielleicht einem oder zwei Mitarbeitern um eine BBBEE-Zertifizierung bemühen. Bei einem Umsatz zwischen 5 und 35 Millionen Rand (rund 2,2 Millionen Euro) dürfen sie sich vier der sieben Kategorien auswählen, nach denen der Betrieb bewertet werden soll. Bei Nichtangabe einer Präferenz berechnet das System die Kategorien mit der besten Wertung. Hierzu muss der Unternehmensinhaber aber die Daten und Kennziffern seines Unternehmens online eingeben. Danach wird der BBBEE-Punktwert auf der BEE-Scorecard errechnet (bester Wert 1,0, schlechtester Wert 8,0). Bewerber in öffentlichen Ausschreibungen (Tenderverfahren) müssen einen guten BBBEE-Score vorweisen können.

In der Wirtschaft nimmt die Kritik am BEE immer weiter zu. Selbst dem COSATU scheint es nicht geheuer, dass sich, offenbar außer einer kleinen schwarzen Elite meist sehr ANC-naher Menschen, die breite Masse der Schwarzen nicht für das Programm begeistern lässt, und schon wieder nach der rassischen Herkunft entschieden werde und auch noch bestimmte Quoten bei Aufträgen für öffentliche Unternehmen eingehalten werden müssten, um mehr und bessere Aufträge zu erhalten. Vor allem wird aber kritisiert, dass Arbeitnehmer nicht, wie im gesamten globalen Wirtschaftssystem üblich, nach Können und voraussichtlichem Nutzen für das Unternehmen eingestellt werden sollen, was zu einem „brain drain“ füh$\mathrm{re}^{532}$. Zudem falle es gnerell schwer, überhaupt qualifizierte Fachkräfte zu finden,

530 Für die bei der Erstellung der Tabelle herangezogenen Daten vgl. o.V. (2015), S. 2; vgl. auch Cargill (2010), S. 235-241, insbesondere für eine Zusammenfassung der BEE-Vorschriften.

531 Anfang 2019 liegt der Wechselkurs des Euros zum Rand bei rund 1:16. Mithin entspricht ein Jahresumsatz von 5 Millionen ZAR rund (der Wechselkurs ändert sich täglich) etwa 310.000 Euro.

532 Vgl. Kiwitter (2015), S. 1. 
gleich ob heller oder dunkler Hautfarbe, was gerade auch ausländischen Investoren Probleme zu bereiten scheint.

Es ist schon interessant, dass das Black Economic Empowerment nicht nur nach Ansicht der Unternehmen ${ }^{533}$ und Handwerksbetriebe, sondern auch nach Meinung des ANC selbst nicht zu deren Zufriedenheit funktionierte, zumal es ungewollt anscheinend sogar die Korruption förderte ${ }^{534}$.

Tatsächlich bestätigten führende Vertreter des ANC und auch ehemalige Minister ${ }^{535}$ schon 2007 in aller Öffentlichkeit, dass das Black Economic Empowerment (BEE) gescheitert sei. Eine gezielte positive Diskriminierung sei nicht mehr zu rechtfertigen und die Politik der Affirmative Action in Südafrika sei fehlgeschlagen. Aus sozialistischer Sicht sei sie ebenfalls, zumal hier das BEE als „crony capitalism“ und sogar als ein Beispiel für eine erfolgreiche „Neo-Apartheid“ (unter dem ANC!) "to co-opt black surrogates" betrachtet wird. ${ }^{536}$

In vielen Ländern der Welt, in denen frühere „Minderheiten“ oder auch bisher unterdrückte „Mehrheiten“ privilegierte Zugänge zu Berufen und bessere Beförderungschancen im Sinne einer auf ausgleichende Gerechtigkeit abzielenden "Affirmative Action“ erhalten, wie z. B. beim Black Economic Empowerment (BEE) oder bei dem $\mathrm{BBBEE}^{537}$, gibt es selbstverständlich eine Unzahl restlicher, nicht einer „Minderheit“ angehöriger Menschen, die sich nun benachteiligt fühlen oder fühlen könnten.

Seit der Abschaffung der Apartheid betonen in bizarrer Weise auch die Farbigen und Asiaten, vor allem die Kap-Malaien oder Inder, immer häufiger, dass man früher zu Zeiten der Apartheid "nicht weiß genug“" („not white enough") gewesen sei, und nun unter der ANC-Regierung, „nicht schwarz genug“" („not black enough“) wäre, um in den Genuss von Affirmative Action-Förderungen aller Art gelangen zu können, wie z. B. dem BEE oder dem BBBEE. ${ }^{538}$

533 Hinzu tritt auch deren Furcht vor Quoten jeder Art, weil man das ,right of management to manage" (Levy (1997), S. 321) aufrechterhalten möchte, und zwar gerade auch im für jeden Betrieb wichtigen Human Resources Management.

534 Vgl. hierzu auch die abschließenden Ausführungen zur Affirmative Action und zum BBBEE im Abschnitt 7.1.

535 So zum Beispiel Alec Erwin, Minister of Public Enterprises.

536 Vgl. Gentle (2007), S. 134 und Turok, Chang \& Feraz (2011), S. 145-159.

537 Für viele rechtliche, wirtschafts- und verfassungsrechtliche und internationale rechtsvergleichende Aspekte zu den Themata „Affirmative Action“ und „BBBEE“, die nicht Thema dieser Untersuchung sein können, vgl. z. B. Gas (2002) und Schneider (2008). Für eine wirtschaftstheoretisch-mikroökonomische Untersuchung zur Politik der Affirmative Action vgl. z. B. Black (1993) und Black (1996).

538 Vgl. Jansen (2009), S. 233-234. 


\subsection{Zum Ende der Ära Mbeki}

\subsubsection{Konzentration auf eindeutige und wachstumsorientierte Programme}

Obgleich das RDP in allen offiziellen Reden und Verlautbarungen stets als Eckstein des wirtschafts- und sozialpolitischen Umbaus und der Weiterentwicklung Südafrikas hervorgehoben wurde, ist das RDP, jedenfalls in seiner Eigenschaft als alleiniges bzw. allein maßgebliches Programm des Umbaus und der Entwicklung Südafrikas, zunehmend infrage gestellt bzw. ja schon seit Jahren durch das GEAR-Programm „ergänzt“ worden.

Mit der zunehmenden Infragestellung des RDP wie des GEAR-Programmes, vor allem mit Verweis auf eine zu geringe Wirksamkeit bei der Armutsbekämpfung, Arbeitsplätzebeschaffung bzw. beim Abbau der Arbeitslosigkeit, durch immer mehr Einzelmitglieder der Tripartite Alliance (ANC-COSATU-SACP) - und sehr ähnlich sahen es langjährige, im Lande lebende Beobachter südafrikanischer Verhältnisse ${ }^{539}$ - begann der Einfluss Präsident Mbekis auf die wirtschafts- und sozialpolitische Orientierung des Landes erkennbar zu sinken.

Fast zum Ende des ersten Jahrzehnts einer ANC-Regierung begann sich Präsident Mbeki darüber öffentlich zu ärgern, wofür Südafrika schon während der Apartheidszeit bekannt war, nämlich für das immer noch erkennbare Nebeneinanderherbestehen einer (früher weißen und marktwirtschaftlichen) Volkswirtschaft der Ersten Welt (,first world economy") und einer mit ihr nicht wirklich verbundenen und marginalisierten Wirtschaft der (früher schwarzen und teils planwirtschaftlichen) Dritten Welt („third world economy $)^{540}$. Hier sollte endlich eine Art Brücke gebaut werden, was in Form eines neuen Wachstumsprogrammes auch bald geschehen sollte. ${ }^{54}$

Bereits im Februar 2006 wurde GEAR durch das Programm Accelerated and shared growth for South Africa (AsgiSA) ${ }^{542}$ mit dem ausdrücklich erklärten Ziel

539 Vgl. z. B. Böhler (2011), S. 75.

540 Mit sehr ähnlichen Worten hatte dies dreizehn Jahre vorher, noch zu Beginn der Regierungszeit Präsident de Klerks, auch der Dekan der wirtschaftswissenschaftlichen Fakultät (Department of Economics) der Universität von Pretoria, Prof. G. L. de Wet "South Africa's two economic systems" (vgl. de Wet (1990), S. 91 und auch Rudman (1991), S. 1-8) beschrieben. Daran schien sich nach fast zehn Jahre RDP einer ANC-Regierung recht wenig geändert zu haben.

541 Vgl. hierzu Gelb (2010), S. 52 und die dort angegebene Literatur.

542 Vgl. für einen ersten Überblick den FOKUS Südafrika, Ausgabe 03/06: „Mehr Wachstum und Gerechtigkeit? Die neue wirtschaftspolitische Initiative Accelerated and Shared 
veröffentlicht, das Programm GEAR zu ergänzen und zu verstärken: „If GEAR was the motor of government economic policy, AsgiSA was to be the turbocharger" ${ }^{\text {"543 }}$

Mit AsgiSA sollte nun endlich die Armut bis 2010 wirksam und spürbar reduziert werden, unter anderem durch Reduzierung, ja Halbierung einer Arbeitslosenquote von über 28 Prozent des Jahres 2004 (und gleichzeitig auch der große Mangel besser und adäquater ausgebildeter Arbeitskräfte) ${ }^{544}$ auf nur mehr 14 Prozent bis zum Jahre 2014. Ganz grundsätzlich betrachtet basiert das Programm auf den wichtigsten Zielen des RDP und sollte für die Errichtung einer vereinigten, demokratischen, nicht sexistischen und nicht rassistischen integrierten Volkwirtschaft sorgen ${ }^{545}$.

In zusammenfassender und vereinfachender Betrachtungsweise könnte man die drei Programme RDP, GEAR und AsgiSA so voneinander abgrenzen, dass das unter Mandela noch allein bestehende, redistributiv und "pro poor" orientierte (aber in einem noch zu frühen Stadium der Transformation noch „nicht zu bezahlende“) RDP nach wiedererfolgter Reintegration Südafrikas in die Weltwirtschaft durch das Programm GEAR zur Erreichung einer makroökonomischen Stabilität ergänzt und unterfüttert wurde. Gleichzeitig hatte sich die Regierung ab 1999 wieder mehr in Richtung "pro poor-Politik“ gewendet, um sich mit der Einführung des AsgiSA ab 2006 verstärkt staatlichen, eher keynesianisch orientierten Investitionen zuzuwenden und auch innerhalb der Tripartite Alliance wieder einen wirtschaftspolitischen Konsens im Sinne eines „post-GEAR consensus ${ }^{\text {“546 }} \mathrm{zu}^{\mathrm{z}}$ erreichen. ${ }^{547}$

Das Programm AsgiSA klärte zunächst über sechs wichtige Umstände und Probleme auf, die das Wirtschaftswachstum in Südafrika hemmen oder einschränken würden:

- Kosten, Effizienz und Kapazität des südafrikanischen logistischen Systems

- rechtliche und sozioökonomische Eintrittsbarrieren, Wettbewerbsbeschränkungen und fehlende Investitionsmöglichkeiten

- mangelnde Ausbildung und fehlende Fachkräfte

Growth Initiative for South Africa (AsgiSA)“. Für den Wortlaut des vollständigen Dokumentes vgl. The Presidency of the Republic of South Africa (2008a) und auch The Presidency of the Republic of South Africa (2008b).

543 Pottinger (2008), S. 78. AsgiSA wurde im Februar 2006 von der damaligen Vize-Präsidentin der Republik Südafrika, Phumzile Mlambo-Ngcuka, lanciert.

544 Vgl. auch Aron (2011), 162.

545 SAHO (2017b), S. 1.

546 Gentle (2009), S. 67.

547 Vgl. ebd., S. 67-68 und Gentle (2007), S. 
- Überregulierung und nötige „deregulation“, vor allem im Hinblick auf kleinere und mittlere Unternehmen (small and medium-sized enterprises, SMEs) ${ }^{548}$

- Defizite der staatlichen Verwaltung und der Führungsqualitäten auf der Leitungsebene

- Volatilität der Währung (starker Kursverfall und starke Kursschwankungen des südafrikanischen Rands (ZAR).

Das zunächst auf 18 Monate angelegte Programm AsgiSA wurde im November 2007 um weitere 18 Monate verlängert und mit diversen weiteren Programmen verbunden, vor allem solchen, die besonders die südafrikanische Industrie fördern sollten, wie z. B. ein Motor Industry Development Programme (MIDP), Automotive Production and Development Plan (APDP, ab 2009 ein bis 2020 konzipiertes Automotive Production and Development Programme mit gleicher Abkürzung und de facto den gleichen Zielen).

Bei den Vorzeigeprojekten bzw. „showpieces in South Africa's post-apartheid industrial strategy ${ }^{\text {“549 }}$ handelt es sich um kapital- und energieintensive Großprojekte („mega projects"), deren Betreiber mit großzügigsten Subventionen, Steuerbefreiungen und erleichterten Zugängen zu Krediten unterstützt werden, und zwar trotz deren eher dürftigen Potentials, neue Arbeitsplätze für Südafrikaner in Südafrika zu beschaffen und eher dazu nützen, ausländische bzw. multinationale Konzerne und deren Anteilseigner „bei der (Fahnen-) Stange zu halten“, was vor

548 Überhaupt war das Thema Existenzgründungsförderung auch kleiner Unternehmen und sogar im informellen Bereich inzwischen „wiederentdeckt“ worden; ein Thema, für welches sich, noch in den 1980er Jahren während der Apartheidszeit, die Regierung nicht zu interessieren schien. Die Initiative ergriff 1979 Anthony Edward Rupert (1916-2006), ein Selfmade-Milliardär, der nach Anfängen mit Zigarettendrehen in einer Garage einen Tabak- und Industriekonzern (Rembrandt Group) errichtet hatte und 1981 die Small Business Development Corporation (SBDC), eine Organisation ohne Gewinnerzielungsabsicht, die mit ihren Existenzgründungsdarlehen seit 1981zur Schaffung mehrerer Hunderttausend Arbeitsplätze beigetragen hat, gegründet (seit Oktober 1998 „Business Partners Limited“ genannt). Seit 2007 gibt es in Johannesburg auch eine National Small Business Chamber (NSBC), die den Sektor der Small and Medium Enterprises (SME) bzw. SMME (Small, Medium and Micro-Enterprises) in jeder Hinsicht unterstützt und weltweit als eine der am schnellsten wachsenden Organisationen dieser Art mit über 125.000 kleinen und großen (multinationale Konzerne) Partnern gilt (Vgl. NSBC (2019)). Seit 2014 gibt es das Small Business Development-Ministerium. Trotz der „Verschlankung“ der neuen Regierung Ende Mai 2019 von 36 auf 28 Ministerien wurde das Small Business Development-Ministerium, geführt von einer neuen Ministerin, Khumbudzo Ntshavheni, als Nachfolgerin von Frau Lindiwe Zulu, beibehalten (vgl. moneyweb (2019)).

549 Marais (2011), S. 149. 
allem Volks- und Betriebswirte und Steuerrechtler in- und außerhalb Südafrikas teils heftig kritisieren.

Tatsächlich handelte es sich auch um ein groß angelegtes Subventionsprogramm für die Automobilindustrie und deren Zulieferbetriebe, das u. a. insbesondere zwei multinationalen (deutschen) Herstellern von Oberklasse und Luxusautomobilen nützte und dessen arbeitsplatzschaffende Wirkung stark umstritten war. ${ }^{50}$

Kurze Zeit nach der Einführung des Wachstumsprogrammes AsgiSA im März 2006 wurde zur Verbesserung der Qualifikation junger Arbeitnehmer bzw. junger Arbeitsloser für zunächst drei Jahre eine (South African Government's) Joint Initiative on Priority Skills Acquisition (JIPSA) gegründet. Beide Programme gehörten eng miteinander zusammen und hatten zeitweise die gleiche Programm-Direktorin (Porgramme Director) Phumzile Mlambo-Ngcuka, damalige Vizepräsidentin/ Deputy President der Republik Südafrika. ${ }^{551}$

Der trotz dieser Programme fehlende Abbau der Armut und der Arbeitslosigkeit in Südafrika führte zu einem Unmut, der u. a. dafür sorgte, dass der ANC bzw. der Nachfolger Präsident Mbekis, Präsident Zuma ein wohl deshalb so gutes Wahlergebnis erzielte, weil er genau das einzuhalten versprach, was seine Vorgänger zwar versprochen hatten, aber nicht einzulösen vermochten, nämlich den Abbau der Armut und der sozioökonomischen Ungleichheit und die Schaffung von neuen Arbeitsplätzen. ${ }^{552}$

Nur ein gutes Jahr nach dem Rücktritt Präsident Mbekis wurde AsgiSA durch einen neuen Wachstumsplan („National Growth Plan“, NGP), den Präsident Jacob Zuma in seiner Lage zur Rede der Nation 2010 vorgestellt hatte, ersetzt, um damit die immer noch Millionen zählenden Arbeitslosen und Armen im Lande und der in Südafrika sogar noch höher gestiegenen Ungleichheit und Armut Herr zu werden. Auch mit dem neuen National Growth Plan (NGP) sollte die strukturelle Arbeitslosigkeit, die Bekämpfung der Armut und die immer noch zu starke Ungleichheit durch Wachstumsförderung bekämpft werden. ${ }^{553}$

Doch auch der NGP gibt kaum klare zeitliche Vorgaben oder wirklich konkrete Umsetzungspläne vor, so dass es im wesentlichen tatsächlich bei gut gemeinten Absichtserklärungen bleibt. Das Wort „entrepreneur", d.h. der dynamische Unternehmer, der vielleicht für Wachstum sorgen könnte, kommt in dem gesamten

550 Vgl. Flatters (2005), S. 17, Marais (2011, S. 149) und auch Bronkhorst, Steyn \& Stiglingh (2013), S. 1296.

551 Seit 2013 Exetutive Director of UN Women (UN Entity for Gender Equality and Empowerment of Women).

552 Vgl. auch Gelb (2010), S. 57.

553 Vgl. auch SAHO (2017b). 
Dokument nur einmal vor ${ }^{554}$, weshalb man den NGP tatsächlich auch als einen Schritt in Richtung einer vielleicht gewollten Zentralisierung mit einer stärkeren staatlichen Steuerung und Staatsintervention deuten kann. ${ }^{555}$

2013 stellte die Regierung noch einen weiteren neuen National Development Plan (NDP) vor ${ }^{556}$, der offenbar auch als strategisch orientierter sozioökonomischer Entwicklungsplan (bis 2030) gedacht war. Auch mit diesem neuen Plan, der wieder Wachstumshemmnisse aller Art benennt, soll eine "more inclusive economy“ angestrebt und bis 2030 die Armut eliminiert und die Ungleichheit behoben und höhere Investitionen, eine Verbesserung der Bildungs- und Berufsausbildungschancen („vocational training") und wachsende Exporte und Entbürokratisierungsbemühungen (zur "deregulation“) ermöglicht werden.

Der weiterhin vielen Schwierigkeiten und Dilemmata der Wirtschafts- und Sozialpolitik im Neuen Südafrika war man sich beim neuen NGP aber immerhin bewusst, wie die folgende Abbildung aus dem NGP klar zeigt (vgl. hierzu Abbildung 2).

Figure 1: The quandary of growth and job creation
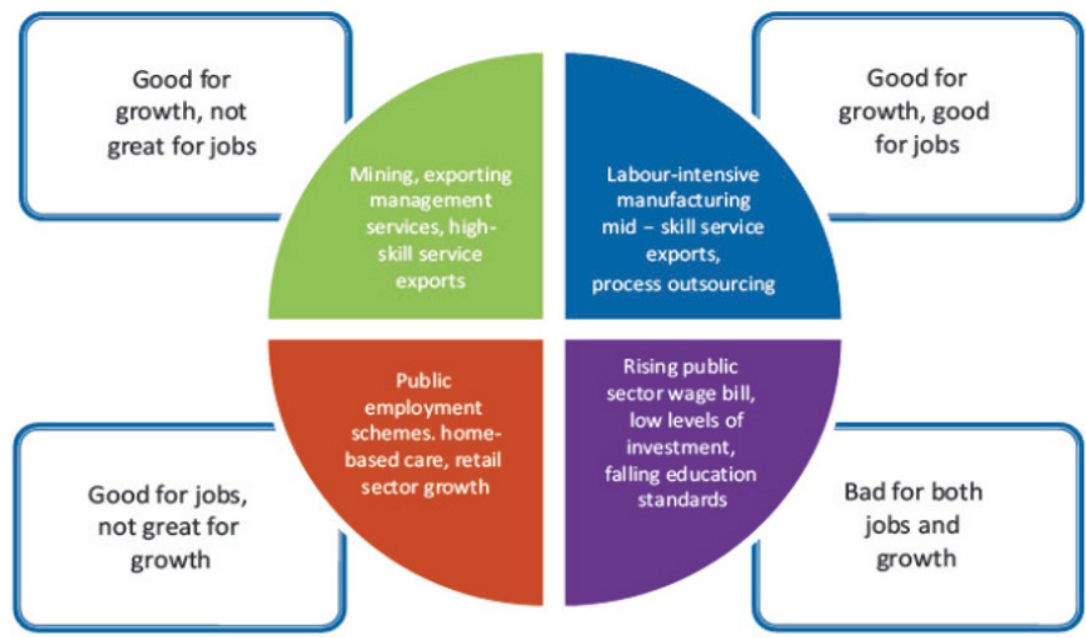

Abb. 2 Dilemmata - Wirtschaftswachstum Arbeitsplätzeschaffung ${ }^{557}$

554 Vgl. Böhler (2011), S. 77.

555 Vgl. ebd., S. 80-82.

556 Vorgestellt von Trevor Manuel, damals Minister in The Presidency: National Planning Commission.

557 Quelle: Politicsweb (2011); S.2. 
Einen großen Strich durch die wirtschaftspolitische Rechnung der südafrikanischen Regierung zogen, auch dies gilt es zu berücksichtigen, diverse internationale Beeinflussungsfaktoren, gerade auch außenwirtschaftliche, wie z.B. die Weltwirtschaftskrise 2008-2016 (siehe auch oben Abschnitt 4.5.1). Doch die Banken-, Staatsverschuldungs- und Weltwirtschaftskrise 2008-2016 kam in der Republik Südafrika allerdings erst nach dem Rücktritt Präsident Mbekis richtig bzw. in Wirtschafts- und Konjunkturdaten erkennbar zur negativen Geltung

Störend auf die Importe wie die Exporte Südafrikas wirkte auch die jahrelang sehr sprunghafte und in der langfristigen Tendenz sehr negative Entwicklung der südafrikanischen Landeswährung Rand (ZAR), die erst in den letzten Jahren, etwa seit dem stärkeren Abschwächen der Weltwirtschaftskrise im Jahre 2016 etwas zur Ruhe kam (siehe hierzu die unten folgenden Abbildungen 3 und 4).

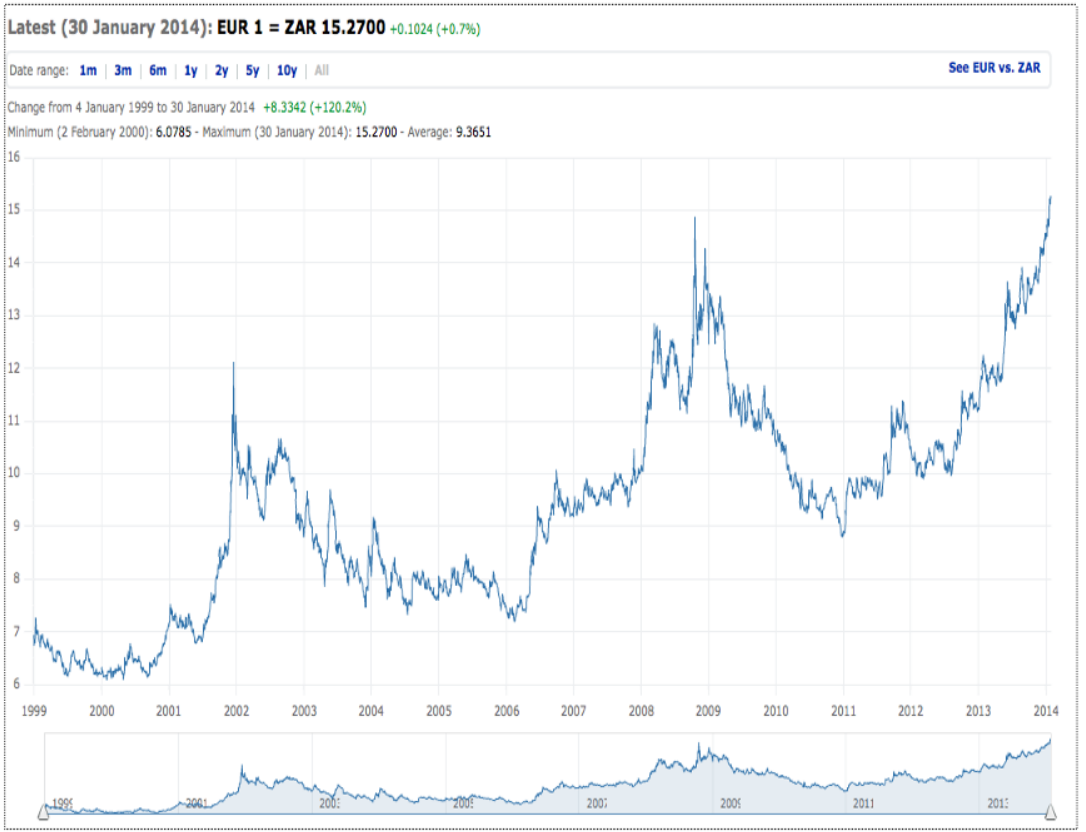

Abb. 3 Entwicklung des Wechselkurses Euro (€) - Rand (ZAR) 1999-2014 558

558 Quelle: Kapstadt entdecken (2019), S.2. 
In Abbildung 4 ist gut zu erkennen, dass bei sinkendem Wechselkurs des südafrikanischen Rand auch das Volumen des südafrikanischen Außenhandelsvolumens (der aus Importen und Exporte in US-Dollar gewogene "trade basket") jeweils in gleicher Richtung sinkt et vice versa.

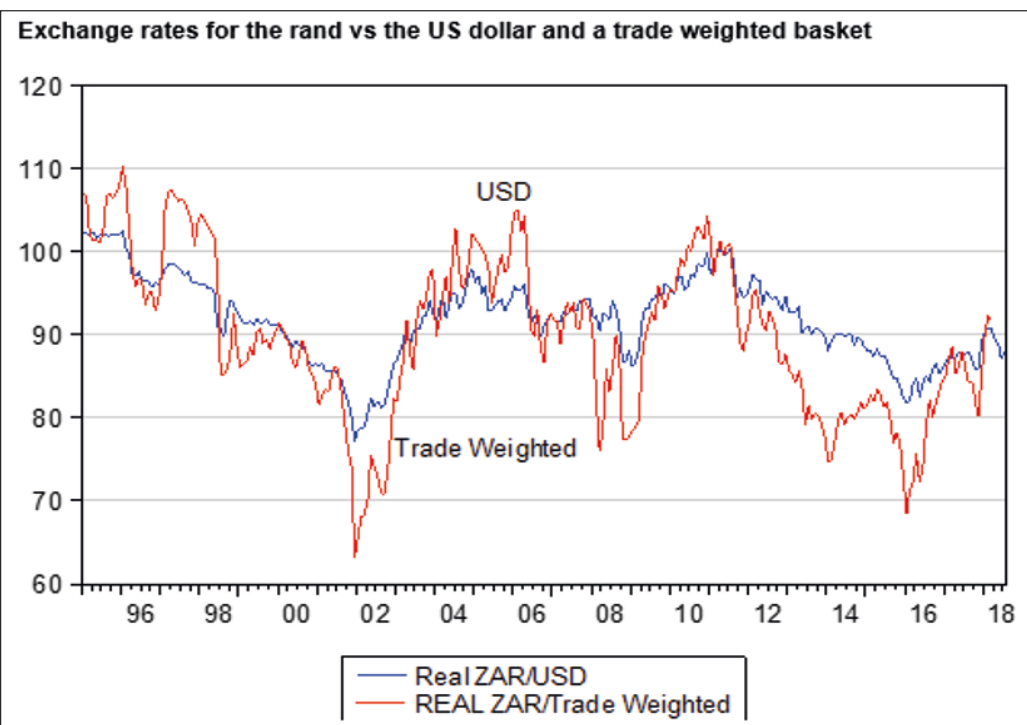

Source: SA Reserve Bank, Federal Reserve Bank of St. Louis and Investec Wealth \& Investment

Abb. 4 Außenhandels- und Wechselkursentwicklung ZAR-US-\$ - 1995-2018559

\subsubsection{Sozioökonomische und politische Gesamtlage zum Ende der Ära Mbeki}

Nach einem insgesamt nur schwächlichen, aber dennoch 16 Jahre ununterbrochenen Wirtschaftswachstum in Südafrika ${ }^{560}$, geriet die Wirtschaft der „Rainbow Nation“ in ihre erste Rezession.

Präsident Mbeki überließ seinem Nachfolger Jacob Gedleyihlekisa Zuma (Präsident vom 9. Mai 2009 bis zum 14. Februar 2018) nicht freiwillig das Ruder.

559 Quelle: Kantor (2018), S.1, vgl. auch Kantor (2015).

560 Vgl. auch Draper, Sidiropoulos \& Leicher (2010), S. 130. 
Vielmehr wurde er zum Rücktritt genötigt, was man sich noch Wochen vorher, nach einer von Mbeki erfolgreich geleiteten ANC Policy Conference in Midrand (Generalthema in der Formulierung des Parteisprechers Smuts Ngonyama "to take

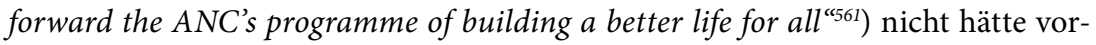
stellen können. Wenige Monate später wurde er, auf dem nationalen Parteitag des ANC Ende 2007 in Polokwane ${ }^{562}$ zunächst als ANC Präsident zugunsten Jacob G. Zumas (2.329 Stimmen, und nur 1.505 für den amtierenden ANC-Präsidenten und Präsidenten der Republik Südafrika, Mbeki) abgewählt. Die ANC-Führung wurde in Polokwane personell erneuert bzw. fast völlig ausgetauscht (in der Hauptsache durch Gefolgsleute Zumas zu Lasten früherer Mbeki-Gefolgsleute) und Präsident a.D. Nelson Mandela hatte in einer schriftlichen Botschaft an die Delegierten des Parteitages seinen „Schmerz“ über die Konflikte, die auf diesem Parteitage zutage getreten waren ${ }^{563}$, einer Partei, die kurz vor Ihrer Spaltung schien, kundgetan.

Im Jahr darauf wurde Mbeki vom Nationalen Exekutiv-Komitee (NEC) des ANC - weniger als ein Jahr vor dem regulären Ablauf seiner Amtszeit (Mai 2009) - zum Rücktritt genötigt. Da Zuma noch nicht Präsident werden konnte (er war zu diesem Zeitpunkt nicht „Member of Parliament“), wurde mit Präsident Motlanthe ein treuer Gefolgsmann Zumas für die Zeit bis zu den Wahlen 2009 zum Präsidenten gewählt, obgleich völlig klar war, dass er diese Funktion nur bis zum erwarteten nächsten Wahlsieg des ANC wahrnehmen würde. Danach hat Motlanthe unter Zuma noch bis 2014 als Vizepräsident („Deputy President $\left.{ }^{\prime \prime}\right)$ gedient. ${ }^{564}$

2005 hatte Mbeki seinen damaligen stellvertretenden Staatspräsidenten Jacob Zuma nach Korruptions- und Vergewaltigungsvorwürfen und gerichtlichen Anklagen aus dem Amt entlassen. Viele Parteigenossen des ANC mit ihm, trotz seiner vergleichsweise disziplinierten und auf Geldwertstabilität bedachten makroökonomischen Politik, die aber zu einem Verlust vieler Arbeitsplätze im formellen Sektor geführt hatte, äußerst unzufrieden. Ganz anders als dem jovialen und lebenslustigen Jacob Zuma („100\% Zulu Boy ${ }^{{ }^{\prime 565}}$ ). Mbeki gelang es nicht. wie Zuma, die Herzen

561 Mail \& Guardian (2007); vgl. auch FOKUS Südafrika, Ausgabe 04/07: „ANC Policy Conference“.

562 Polokwane ist die Hauptstadt der Provinz Limpopo und liegt etwa 250 km nordnordöstlich von Pretoria.

563 Vgl. „Un leader zulu per il partito di Mandela“ in La Repubblica vom 19.08.2007.

564 Vgl. Pollak (2009), S. 165, und auch Sidiropoulos (2009), S. 49-50.

565 So zu lesen auf einem Plakat eines Zuma-Anhängers, der mit Tausenden anderer Anhänger Zumas während eines Prozesses anlässlich einer Anklage gegen Jacob Zuma wegen Vergewaltigung der Tochter eines verstorbenen Parteigenossen, die HIV-positiv war, vor dem Gerichtsgebäude protestiert hatte (vgl. Niehaus (2010), S. 157, dort auch das wörtliche Zitat). Der damals 64jährige Zuma, übrigens mehrfach verheiratet, selbst 
der Menschen, besonders aber die seiner Parteigenossen zu gewinnen, und sie von seiner Politik zu überzeugen, was ihn schließlich sein Amt gekostet hat.

2008, im letzten Regierungsjahr Mbekis, und fast Jahre 15 nach der Einführung des RDP und zum nahenden Ende der 3. Legislaturperiode, stellte zum Beispiel auch das südafrikanische Büro der Friedrich-Ebert-Stiftung, welche den ANC seit Anfang der 1990er Jahre stets wohlwollend begleitet und unterstützt hat, fest, dass immer noch ein Drittel der Südafrikaner ,in Armut“ lebt. ${ }^{566}$

Besonders verärgert war die „ANC-Basis“ wegen der allesamt kontraproduktiven Politik der (forcierten) affirmative action (BEE und BBBEE), wegen der fehlenden Armutsbeseitigung, wegen Mbekis Simbabwepolitik ganz sicher auch - ohne dies angesichts wohl sieben Millionen AIDS-infizierter Südafrikaner ${ }^{567} \mathrm{zu}$,laut " zu sagen oder zu schreiben - wegen seiner Leugnung der Gefährlichkeit und des Ausmaßes des AIDS-Problems bzw. wegen seiner völlig missglückten AIDS-Politik; dies vor dem Hintergrund der menschlichen, medizinischen, politischen und sozioökonomischen Katastrophe, welche die AIDS-Pandemie in Südafrika und im ganzen südlichen Afrika hervorgerufen und damit auch die sozioökonomische Transformation zum Neuen Südafrika um Jahre zurückgeworfen haben dürfte, zumal die HIV/AIDS-Infektionsrate im südlichen (Subsahara-) Afrika schon 2001 etwa sechs bis sieben Mal so hoch wie in den südlichen Entwicklungsländern insgesamt lag. ${ }^{568}$

gab zu, „ungeschützten“ Sexualverkehr mit der 3ljährigen HIV-positiven jungen Frau gehabt zu haben und sich danach („, to cut the risk of contracting HIV“ (ebd.)), lediglich geduscht zu haben. Während der Prozesspausen sang Zuma auf isiZulu: „Umshini wami, Umshini wami, We Baba. Awuleth, umshini wami" (Mein Maschinengewehr, mein Maschinengewehr! O, Vater („Papa“)! Bitte bring mir mein Maschinengewehr!) und tanzte gemeinsam mit dem Heer der Demonstranten vor dem Gerichtsgebäude. Zuma wurde 2006 von Richter Willem van der Merwe als nicht schuldig im Sinne der Anklage (mangels das Gericht vollständig überzeugender Beweise) freigesprochen, aber wegen seines leichtsinnigen bzw. fahrlässigen („reckless behaviour") Benehmens bzw. Verhaltens gerügt (vgl. ebd.). Jahre später kritisierte der Verfassungsrichter a.D. Zak Jacoob öffentlich das Urteil und verkündete, dass er selbst Zuma wegen Vergewaltigung verurteilt hätte (vgl. iol (2014)). Zu Details des Verfahrens vgl. Mail \& Guardian (2010) und für einen kurzen Filmbericht mit beeindruckenden Bildern aus dem Gerichtssaal und von den vielen Demonstranten vor dem Gerichtsgebäude vgl. AParchive/youtube (2015)).

566 FES-Büro Südafrika (2008), S. 3.

567 UN-AIDS ging und andere Quellen gingen 2005/2006 von 5,7 Millionen (vgl. auch Kadalie (2008) (,,̈̈ber fünf Millionen“), und Sandfort, Reddy \& Rispel (2009b)) und für 2014 von etwa 6,8 Millionen aus (vgl. UN AIDS (2015a) und UN AIDS (2015b)) für sämtliche exakten Zahlen für Südafrika und andere Länder.

568 Vgl. Eberlei (2009), S. 56, und auch dort angegebene Quellen. 
Zehn Jahre nach Einführung des GEAR-Programmes bewegten sich in der zweiten Hälfte der zweiten Amtszeit Präsident Mbekis die Arbeitslosenquoten immer noch zwischen etwa 30-40 Prozent, und die wahrzunehmende Ungleichheit im Lande hatte weiter zugenommen. Nachdem GEAR ein halbes Jahrzehnt benötigt hatte, um erkennbare Wirkungen zu zeigen, wuchs die Wirtschaft zwischen 2004 und 2007 endlich um etwa 4-5,5 Prozent an (siehe Abbildung 1 am Ende des Abschnittes 4.5.2), um jedoch mit Beginn der Weltwirtschaftskrise schon wieder auf nur noch gut 3 Prozent zu sinken, im Jahr 2008 sogar zu schrumpfen (fast 2 Prozent Minus beim BIP) und sich erst ab 2009 wieder ins Plus zu begeben. Das kleine Plus verblieb die ganzen nächsten Jahre stets unter der von Volkswirten stets erwünschten Mindestwachstumsrate des BIP von 6 Prozent, welche als Minimum für eine verstärkte und nachhaltige Schaffung von Arbeitsplätzen betrachtet wurden.

Bis zum September 2008 schien das Zuma-Lager im ANC noch eine eher amorphe, dabei jedoch immer größer werdende Koalition von Mbeki-Gegnern zu sein, die aus Mitgliedern der unzufriedenen Basis, eher militanten Gruppen und der Parteilinken (ANC Youth League, ANC Women's League (ANCWL)), COSATU und SACP) unterstützt wurden. Sie wurde derart stark, dass Mbeki de facto vom NEC gestürzt werden konnte und zum Herbst 2008 seinen Rücktritt erklären musste ${ }^{569}$.

Ausgerechnet Jacob Zuma, den Mbeki wegen Korruptions- und anderer Vorwürfe 2005 aus dem Amt des Vizepräsidenten entlassen hatte, wurde nach den wenigen Monaten Regierungszeit Präsident Motlanthes, sein Nachfolger.

Mbekis Nachfolger, Präsident Jacob G. Zuma, den Pollak drastisch als „praktizierenden Polygamisten mit rudimentärer Bildung, der unter Korruptionsverdacht stand und eine Vergewaltigungsanklage nur aus verfahrensrechtlichen Gründen abschütteln konnte ${ }^{\text {"570 }}$ beschreibt, hatte die wahrlich wenig rühmliche AIDS-Politik bzw. eine AIDS zumindest verharmlosende, die Gefährlichkeit und Mortalitätsraten der Infektion bzw. den Zusammenhang zwischen HI-Virus und AIDS leugnende und anfänglich gar nicht wahrhaben wollende (oder AIDS nicht verstehende) Politik („aids denial“) seines Vorgängers Mbeki ${ }^{571}$ de facto lange Zeit auch noch

569 Vgl. Pabst \& von der Ropp (2009), S.71-72.

570 Vgl. ebd. bzw. Pollak (2009), S. 165-166, in Übersetzung des Verfassers.

571 Um der Gerechtigkeit willen muss aber auch Russel zitiert werden, der von Mbekis "aids denial“ schreiben konnte bzw. musste: „The fatalism surrounding discussion on AIDS cannot all be blamed on Mbeki. He was preaching his denialist message to a receptive audience" (Russel (2010), S.227). Auch in vielen anderen Ländern der Erde, selbst in der Volksrepublik China, gab es lange Zeit ein Stillschweigen und Leugnen der HIV/AIDS-Krise. Erst zu ähnlicher Zeit wie in Südafrika begann man, anscheinend stimmige und ehrliche Zahlen zu veröffentlichen und das Problem wirklich landesweit anzugehen (vgl. hierzu z. B. Pisani (2009), S. 318-324). 
fortgesetzt ${ }^{572}$. Allerdings wurden schon im Jahr nach seinem Dienstantritt vom Department for Social Development 58 Prozent mehr Hilfsstellen für Menschen gebaut, die mit einer HIV-Infektion leben müssen ${ }^{573}$, nachdem die Mittel für die Infrastruktur schon Ende 2008 kräftig erhöht worden waren. ${ }^{574}$

Dies alles, obwohl die vielen Sterbefälle (etwa 7 Millionen aktuell infizierte HIV-positive Menschen Südafrikas ${ }^{575}$ ) nicht nur zu Arbeitsausfällen ${ }^{576}$, gewaltigen medizinischen Kosten etc. führten, sondern weil es zu gewaltigen Ausfällen des in Südafrika dringend benötigten Bruttoinlandsproduktes bzw. dessen Steigerungen gekommen ist ${ }^{577}$. Die Millionen bis Billionen Rand, welche diese menschliche und gesamtgesellschaftliche Katastrophe gekostet hat, sind kaum korrekt zu ermitteln ${ }^{578}$, doch existierten schon vor etwa 15-16 Jahren Schätzungen darüber, dass Südafrikas Wirtschaftskraft im zweistelligen Bereich schrumpfen dürfte. ${ }^{579}$

572 Als Präsident a.D. Jacob Zuma, der gerade wegen Vergewaltigung einer an HIV-positiven Frau vor Gericht stand, befragt wurde, ob er sich der Ansteckungsgefahren bewusst sei, antwortete Zuma öffentlich dass er „danach“ eine Dusche genommen habe, um sich selbst gegen eine HIV-Infektion zu schützen (vgl. z. B. Nattrass (2011), S. 194, und viele andere mögliche Quellen). Einzelne Karikaturisten zeichneten Präsident Zuma in den Jahren danach oft mit einem aus dem hinteren Teil des Schädels „herauswachsenden“ Dusch- bzw. Brausekopf, der wie eine Art unechter Heiligenschein („halo“) über seinem Haupte montiert zu sein schien.

573 So der tägliche amtliche Nachrichtendienst „BuaNews online“ (heute SAnews) in der Ausgabe vom 16.10.2009.

574 Vgl. BuaNews online vom 20.11.2008: „SA committed to increased infrastructure spending“.

575 Es ist bis heute schwierig, exakte Zahlen zu erhalten. Schon vor zehn Jahren wurde die Zahl der infizierten Menschen mit 5,7 Millionen (und eher mehr) angegeben (vgl. Jankowiak (2009), S. 114), der auch darauf hinweist, dass es sich bei Aids nicht nur um eine Epidemie, sondern um eine Pandemie handelt, die „UNO-Diktion“ aber von „Epidemie“ spricht (ebd., S. 113); Vgl. auch Posel (2008)).

576 Vgl. „Gegen den schleichenden Tod in der Belegschaft“, FAZ vom 30.04.2005“, wo beschrieben wird, wie internationale Konzerne in Südafrika mit AIDS umgehen.

577 Vgl. Bigsten \& Durevall (2008), S. 37, und die dort zitierte Literatur.

578 Auch hier wieder wegen fehlender statistischer Erfassung der Patientendaten. Es fiel aber überall in Südafrika auf, dass mehrere hunderttausend Kinder pro Jahr mit der Diagnose „Durchfall“ schon im Kindbett gestorben waren, wovon ein heute kaum mehr bestimmbarer Teil, sehr wahrscheinlich ein größerer Teil, in Wirklichkeit an einer schon im Mutterleib zugezogenen HIV-Infektion gestorben sein könnte.

579 Wittek zitierte hier schon im Jahre 2003 Schätzungen des Aids-Bekämpfungsprogrammes der Vereinten Nationen (Joint United Nations Program on Aids, UN AIDS), nach dem Südafrikas Volkswirtschaft bis 2010 um etwa 17 Prozent schrumpfen würde (vgl. Wittek (2003), S. 153). 
Auf jeden Fall hatte man in der Regierung erkannt, dass der Kampf zur Beseitigung der Armut in Südafrika sich (endlich) erheblich deutlicher im Staatshaushalt widerspiegeln müsse, ja ein „Pro-Poor Budgeting ${ }^{\text {“580 }}$ nötig wäre. Hierzu könne durch Änderungen in der Aufstellung des Staatshaushaltes, d.h. im Rahmen des Budgetierungsprozesses selbst ${ }^{581}$, v. a. durch eine stärkere Beteiligung der (interessierten) Öffentlichkeit ${ }^{582}$ bzw. der Steuerzahler und Wähler beigetragen werden.

Unter Präsident Zuma wurden dann noch diverse weitere wirtschafts- und sozialpolitische, vor allem wieder wachstumsorientierte Programme entwickelt ${ }^{583}$ und teilweise auch umgesetzt, mit denen aber bis 2019 kein hinreichendes und damit auch ein kein adäquates Wirtschaftswachstum erreicht werden konnte.

580 Vgl. Verwey (2009) und Ajam (2009).

581 Vgl. Wehner (2009), S. 31-38, und Mohamed (2009).

582 Vgl. Lefko-Everett \& Zamisa (2009).

583 Vgl. hierzu den von Parsons herausgegebenen Sammelband zu vielen wichtigen sozioökonomischen Aspekten der Wirtschafts- und Sozialpolitik, z. B. zur Arbeitsmarktpolitik, zur Gesundheitspolitik, zur Handels-, Geld- und Währungspolitik schon zu Beginn der Regierungszeit Präsident Zumas (Parsons (2009)). 